\title{
Algebraic varieties on which the classical Phragmén-Lindelöf estimates hold for plurisubharmonic functions
}

\author{
Rüdiger W. Braun ${ }^{1}$, Reinhold Meise ${ }^{1}$, B.A. Taylor ${ }^{2}$ \\ 1 Mathematisches Institut, Heinrich-Heine-Universität, Universitätsstraße 1, 40225 Düssel- \\ dorf, Germany (e-mail: braun@cs.uni-duesseldorf.de / meise@cs.uni-duesseldorf.de) \\ 2 Department of Mathematics, University of Michigan, Ann Arbor, MI 48109, USA \\ (e-mail: taylor@umich.edu)
}

Received July 23, 1998

\begin{abstract}
Algebraic varieties $V$ are investigated on which the natural analogue of the classical Phragmén-Lindelöf principle for plurisubharmonic functions holds. For a homogeneous polynomial $P$ in three variables it is shown that its graph has this property if and only if $P$ has real coefficients, no elliptic factors, is locally hyperbolic in all real characteristics, and the localizations in these characteristics are square-free. The last condition is shown to be necessary in any dimension.
\end{abstract}

Mathematics Subject Classification (1991): Primary 32F05, 31C10

\section{Introduction}

An algebraic variety $V$ in $\mathbb{C}^{n}, n \geq 2$, has the property (SPL) if there exists a constant $A \geq 1$ such that for each plurisubharmonic function $u$ on $V$ the estimates

$$
u(z) \leq|z|+o(|z|), z \in V, \quad \text { and } \quad u(z) \leq 0, z \in V \cap \mathbb{R}^{n},
$$

imply

$$
u(z) \leq A|\operatorname{Im} z|, z \in V .
$$

The third author gratefully acknowledges support of the A. v. Humboldt Stiftung for visits at Mathematisches Institut der Heinrich-Heine-Universität Düsseldorf, where this research was carried out. 
By the classical Phragmén-Lindelöf Theorem, $V=\mathbb{C}^{n}$ satisfies (SPL) with $A=1$. Thus the varieties with the property (SPL) are the ones for which a natural extension of the Phragmén-Lindelöf Theorem holds. This, however, is not the only reason why they are of interest. By results of Meise, Taylor, and Vogt [10], [11], [15], the zero variety

$$
V\left(P_{m}\right)=\left\{z \in \mathbb{C}^{n}: P_{m}(z)=0\right\}
$$

of a homogeneous polynomial $P_{m}$ of degree $m \geq 2$ in $n$ variables satisfies (SPL) if and only if the differential operator $P(D): C^{\infty}\left(\mathbb{R}^{n}\right) \rightarrow C^{\infty}\left(\mathbb{R}^{n}\right)$ admits a continuous linear right inverse. Moreover, results of Hörmander [8] imply that for each $Q \in \mathbb{C}\left[z_{1}, \ldots, z_{n}\right]$ with $\operatorname{deg} Q<m$ the operator $\left(P_{m}+Q\right)(D)$ acts surjectively on the space $\mathcal{A}\left(\mathbb{R}^{n}\right)$ of all real-analytic functions if $V\left(P_{m}\right)$ has (SPL). It is reasonable to ask why $A>1$ is allowed in (1.2). Proposition 2.8 explains why the case $A=1$, while interesting, is too restrictive. For example, $V\left(P_{m}\right)$ satisfies (SPL) with $A=1$ if and only if $P_{m}$ is a product of real linear forms.

To consider non-homogeneous polynomials, let $P_{m} \in \mathbb{C}\left[z_{1}, \ldots, z_{n}\right]$ be homogeneous of degree $m \geq 2$ and define $P \in \mathbb{C}\left[z_{1}, \ldots, z_{n+1}\right]$ by

$$
P\left(z^{\prime}, z_{n+1}\right):=P_{m}\left(z^{\prime}\right)-z_{n+1} .
$$

In this situation, Meise and Taylor [9] have shown recently that if $V(P)$ satisfies (SPL) then $P_{m}$ is square-free and has real coefficients. They also proved that $V(P)$ satisfies (SPL) if $P_{m}$ is of real principal type and none of its irreducible factors is elliptic. For $n=2$ this condition is a characterization and is equivalent to $P_{m}$ being strictly hyperbolic or $P_{m}$ being a product of $m$ distinct real linear forms. Moreover, Meise and Taylor [9] showed that for real homogeneous polynomials $P_{m}, V(P)$ has (SPL) if and only if both varieties

$$
V_{ \pm}\left(P_{m}\right):=\left\{z \in \mathbb{C}^{n}: P_{m}(z)= \pm 1\right\}
$$

have (SPL) and that $V(P)$ has (SPL) if and only if the operator $P(D)$ : $C^{\infty}\left(\mathbb{R}^{n+1}\right) \rightarrow C^{\infty}\left(\mathbb{R}^{n+1}\right)$ admits a continuous linear right inverse.

In the present paper we improve the necessary condition of $P_{m}$ being square-free and use the improvement to characterize in dimension $n=3$ when $V(P)$ has (SPL). To formulate the result, denote by $\left(P_{m}\right)_{\theta}$ the lowest order homogeneous polynomial in the expansion of $z \mapsto P_{m}(\theta+z)$. Using the concept of quasihomogeneity and a result of [4], we show that if $V(P)$ has (SPL) then $\left(P_{m}\right)_{\theta}$ is square-free for each $\theta \in V\left(P_{m}\right) \cap \mathbb{R}^{n},|\xi|=1$. Of course, this condition is a lot more restrictive than the requirement that $P_{m}$ is square-free. In fact, for $n=3$ it leads to the following characterization: 
Theorem 1.1. Let $P_{m} \in \mathbb{C}\left[z_{1}, z_{2}, z_{3}\right]$ be homogeneous of degree $m \geq 2$ and define $P \in \mathbb{C}\left[z_{1}, \ldots, z_{4}\right]$ by $P(z):=P_{m}\left(z_{1}, z_{2}, z_{3}\right)-z_{4}$. The following are equivalent:

(1) $V(P)$ has (SPL),

(2) $P(D): C^{\infty}\left(\mathbb{R}^{4}\right) \rightarrow C^{\infty}\left(\mathbb{R}^{4}\right)$ admits a continuous linear right inverse,

(3) $V_{+}\left(P_{m}\right)$ and $V_{-}\left(P_{m}\right)$ satisfy (SPL),

(4) $V\left(P_{m}\right)$ has $(\mathrm{SPL}), P_{m}$ has real coefficients, and $\left(P_{m}\right)_{\theta}$ is square-free for each $\theta \in V\left(P_{m}\right) \cap \mathbb{R}^{3}$ with $|\theta|=1$.

Examples like Newton's knot and the Cartesian leaf in homogeneous coordinates

$$
P_{3}(x, y, z)=y^{2} z-x(x-z)^{2} \quad \text { and } \quad P_{3}(x, y, z)=x^{3}+y^{3}-3 x y z
$$

satisfy these conditions (see Example 4.11). Hence for $n \geq 3$ there are homogeneous polynomials $P_{m} \in \mathbb{R}\left[z_{1}, \ldots, z_{n}\right]$ for which $V(P)$ has (SPL), while $V\left(P_{m}\right) \cap \mathbb{R}^{n}$ contains non-zero singular points, contrary to the situation for $n=2$.

Because of the results of Meise and Taylor [9], the essential point in proving Theorem 1.1 is to show that (4) implies (3). To do this we apply a recent result of [5], by which there exists a constant $A_{0} \geq 1$ such that each $u \in \operatorname{PSH}\left(V_{ \pm}\left(P_{m}\right)\right)$ which satisfies (1.1) already satisfies

$$
u(z) \leq A_{0}|z|, \quad z \in V_{ \pm}\left(P_{m}\right) .
$$

From this it follows that it suffices to prove the estimate (1.2) for $u$ only at points $z \in V_{ \pm}\left(P_{m}\right)$ for which $|\operatorname{Im} z|$ is small compared to $|z|$. Since both $V_{+}\left(P_{m}\right)$ and $V_{-}\left(P_{m}\right)$ are manifolds, points in a fixed ball of arbitrary size are easy to handle. Since points $z \in V_{ \pm}\left(P_{m}\right)$ with large $|z|$ are close to points in $V\left(P_{m}\right)$, it suffices to consider $V_{ \pm}\left(P_{m}\right)$ in cones around real lines spanned by $\xi \in V\left(P_{m}\right) \cap \mathbb{R}^{3},|\xi|=1$. To handle $V_{ \pm}\left(P_{m}\right)$ in such cones we use a result of Braun [1] by which there exist suitable coordinates so that in some neighborhood of $\xi$ the variety $V\left(P_{m}\right)$ is a finite union of graphs of holomorphic functions which are real-valued for real arguments. Assuming $\xi=(1,0,0)$ it follows from this that for large $t>0$ the varieties $V_{ \pm}\left(P_{m}\right)$ near $(t \xi, 0,0)$ can be approximated sufficiently well by $V_{ \pm}(Q)$, where $Q\left(z_{1}, z_{2}, z_{3}\right)=z_{1}^{m-\nu} Q_{0}\left(z_{2}, z_{3}\right)$ for some $\nu \in \mathbb{N}$ and some $Q_{0} \in$ $\mathbb{R}\left[z_{2}, z_{3}\right]$ which is strictly hyperbolic. Knowing this, we use compactness and scaling arguments together with bounds for particular harmonic functions to derive the estimate (1.2) for $u$ in a cone around $\mathbb{R} \xi$ from the fact that we already know (1.3). 


\section{Preliminaries}

In this section we introduce the basic definitions that will be used subsequently.

Definition 2.1. Let $V$ be an algebraic variety in $\mathbb{C}^{n}$ and $\Omega$ be an open subset of $V$. A function $u: \Omega \rightarrow[-\infty, \infty$ [ will be called plurisubharmonic if it is locally bounded above, plurisubharmonic in the usual sense on $\Omega_{\text {reg }}$, the set of all regular points of $V$ in $\Omega$, and satisfies

$$
u(z)=\limsup _{\xi \in \Omega_{\mathrm{reg}}, \xi \rightarrow z} u(\xi)
$$

at the singular points of $V$ in $\Omega$. By $\operatorname{PSH}(\Omega)$ we denote the set of all plurisubharmonic functions on $\Omega$.

Definition 2.2. Let $V$ be an algebraic variety in $\mathbb{C}^{n}, n \geq 2$, and $A \geq 1$, $B \geq 0$. We say:

(a) $V$ satisfies the condition $\operatorname{SPL}(A, B)$ if for each $u \in \operatorname{PSH}(V)$ the conditions $(\alpha)$ and $(\beta)$ imply $(\gamma)$, where
( $\alpha) u(z) \leq|z|+o(|z|), z \in V$
(ß) $u(z) \leq 0, z \in V \cap \mathbb{R}^{n}$,
( $\gamma) u(z) \leq A|\operatorname{Im} z|+B, z \in V$.

(b) $V$ satisfies the condition (SPL) (the strong Phragmén-Lindelöf condition) if $V$ satisfies $\operatorname{SPL}(A, 0)$ for some $A \geq 1$.

Remark. By the classical Phragmén-Lindelöf Theorem, $\mathbb{C}^{n}$ satisfies $\mathrm{SPL}(1,0)$. Hence the algebraic varieties satisfying (SPL) are those on which the obvious extension of the classical Phragmén-Lindelöf condition holds. To indicate why these varieties are of interest also in a different context, we recall the following notions.

Definition 2.3. Let $\omega:[0, \infty[\rightarrow] 0, \infty[$ be continuous and increasing and assume that it has the following properties:
$(\alpha) \quad \omega(2 t)=O(\omega(t))$
( $\beta) \quad \int_{1}^{\infty} \frac{\omega(t)}{t^{2}} d t<\infty$
( $\gamma) \quad \log t=O(\omega(t))$ as $t$ tends to infinity
( $\delta) \quad x \mapsto \omega\left(e^{x}\right)$ is convex.

Then $\omega: z \mapsto \omega(|z|), z \in \mathbb{C}^{n}, n \in \mathbb{N}$, will be called a weight function. Also we will assume without restriction that $\omega(0) \geq 1$. For examples we refer to [3]. 
Definition 2.4. Let $V$ be an algebraic variety in $\mathbb{C}^{n}$ and $\omega$ a weight function. $V$ satisfies the condition $\operatorname{PL}\left(\mathbb{R}^{n}, \omega\right)$ of Phragmén-Lindelöf type, if the following holds:

There exists $A \geq 1$ so that for each $\rho>0$ there exists $B_{\rho}>0$ so that each $u \in \operatorname{PSH}(V)$ which satisfies $(\alpha)$ and $(\beta)$ also satisfies $(\gamma)$, where

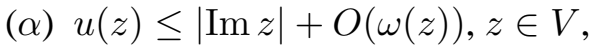

(ß) $u(z) \leq \rho|\operatorname{Im} z|, z \in V$,

$(\gamma) u(z) \leq A|\operatorname{Im} z|+B_{\rho} \omega(z), z \in V$.

If $\omega(t)=\log (2+t)$ then we will write $\operatorname{PL}\left(\mathbb{R}^{n}, \log \right)$.

One relation between the conditions $\operatorname{SPL}(A, B)$ and $\operatorname{PL}\left(\mathbb{R}^{n}, \omega\right)$ is explained in the following lemma.

Lemma 2.5. For each algebraic variety $V$ in $\mathbb{C}^{n}$ the following assertions hold:

(a) If $V$ satisfies $\operatorname{SPL}(A, B)$ for some $A \geq 1, B \geq 0$, then $V$ satisfies $\mathrm{PL}\left(\mathbb{R}^{n}, \omega\right)$ for each weight function $\omega$.

(b) If $V$ is homogeneous and satisfies $\mathrm{PL}\left(\mathbb{R}^{n}, \omega\right)$ for some weight function $\omega$ then $V$ satisfies (SPL).

In particular, $(\mathrm{SPL})$ and $\mathrm{PL}\left(\mathbb{R}^{n}, \omega\right)$ are equivalent if $V$ is homogeneous.

Proof. (a) Since each weight function $\omega$ satisfies $\omega(t)=o(t)$ as $t$ tends to infinity, this is easily checked.

(b) This follows from Meise, Taylor, and Vogt [15], Theorem 3.1, in connection with Meise and Taylor [9], Lemma 3.2.

2.6. Differential operators and Phragmén-Lindelöff conditions. For $P \in$ $\mathbb{C}\left[z_{1}, \ldots, z_{n}\right], P(z)=\sum_{|\alpha| \leq m} a_{\alpha} z^{\alpha}$, we define the differential operator $P(D)$ with symbol $P$ by

$$
P(D):=\sum_{|\alpha| \leq m} a_{\alpha} \frac{1}{i^{|\alpha|}} \frac{\partial^{|\alpha|}}{\partial x_{1}^{\alpha_{1}} \ldots \partial x_{n}^{\alpha_{n}}} .
$$

When $\sum_{|\alpha|=m}\left|a_{\alpha}\right| \neq 0, P$ has degree $m$ and its principal part $P_{m}$ is defined as $P_{m}(z)=\sum_{|\alpha|=m} a_{\alpha} z^{\alpha}$. Sometimes, $P_{m}$ will just denote an arbitrary homogeneous polynomial of degree $m$. We will use the notation

$$
V(P):=\left\{z \in \mathbb{C}^{n}: P(z)=0\right\}
$$

and $V_{ \pm}\left(P_{m}\right):=V\left(P_{m} \mp 1\right)$.

By Meise, Taylor, and Vogt [11], [14], the variety $V(P)$ satisfies $\operatorname{PL}\left(\mathbb{R}^{n}\right.$, $\log )\left(\right.$ resp. $\left.\operatorname{PL}\left(\mathbb{R}^{n}, \omega\right)\right)$ if and only if the differential operator $P(D)$ admits a continuous linear right inverse on $C^{\infty}\left(\mathbb{R}^{n}\right)$ and/or $\mathcal{D}^{\prime}\left(\mathbb{R}^{n}\right)$ (resp. on 
$\mathcal{E}_{(\omega)}\left(\mathbb{R}^{n}\right)$ and/or $\left.\mathcal{D}_{(\omega)}^{\prime}\left(\mathbb{R}^{n}\right)\right)$. Palamodov [16] has shown that the splitting of a differential complex of $C^{\infty}$-functions or distributions over $\mathbb{R}^{n}$ is equivalent to $\mathrm{PL}\left(\mathbb{R}^{n}, \log \right)$ holding for the corresponding varieties.

By Meise, Taylor, and Vogt [15], Theorem 4.1, $V\left(P_{m}\right)$ has $\operatorname{PL}\left(\mathbb{R}^{n}, \log \right)$ and hence (SPL), whenever $V(P)$ satisfies $\operatorname{PL}\left(\mathbb{R}^{n}, \omega\right)$ for some weight function $\omega$. Therefore it is reasonable to treat general differential operators $P(D)$ as perturbations of their principal part $P_{m}(D)$. In this connection, Meise and Taylor [9] showed recently that the condition (SPL) plays an important role when $P_{m}$ is perturbed by an independent variable. From [9], Theorem 3.4, we recall the following theorem.

Theorem 2.7. Let $P_{m} \in \mathbb{R}\left[z_{1}, \ldots, z_{n}\right]$ be homogeneous of degree $m \geq 2$ and let $P(z):=P_{m}\left(z^{\prime}\right)-z_{n+1}$ for $z=\left(z^{\prime}, z_{n+1}\right) \in \mathbb{C}^{n+1}$. Then the following assertions are equivalent:

(1) $V(P)$ satisfies $\mathrm{PL}\left(\mathbb{R}^{n+1}, \log \right)$,

(2) $V(P)$ satisfies $\mathrm{PL}\left(\mathbb{R}^{n+1}, \omega\right)$ for some weight function $\omega$ with $\omega(t)=$ $o\left(t^{1 / m}\right)$

(3) $V_{ \pm}\left(P_{m}\right)$ both satisfy (SPL),

(4) $V(P)$ satisfies $(\mathrm{SPL})$,

(5) $V(P)$ satisfies $\mathrm{PL}\left(\mathbb{R}^{n+1}, \omega\right)$ for each weight function $\omega$.

It is natural to ask why we consider $\operatorname{SPL}(A, 0)$ for constants $A>1$. The case $A=1$ is also interesting but it is very restrictive. For example, there are no polynomials of degree $m>1$ with an irreducible principal part that can satisfy SPL with $A=1$. To explain this in a bit more detail, we include the following proposition that is essentially well-known.

Proposition 2.8. Let $P \in \mathbb{C}\left[z_{1}, \ldots, z_{n}\right]$ be of degree $m \geq 1$ and denote by $P_{m}$ its principal part. If $V(P)$ satisfies $\mathrm{SPL}(1,0)$ then $P$ and $P_{m}$ are hyperbolic with respect to each vector $N \in \mathbb{R}^{n} \backslash\{0\}$ satisfying $P_{m}(N) \neq 0$. In particular, up to a complex factor, $P_{m}$ is the product of $m$ real linear forms.

Proof. For $\rho>0$ let $K_{\rho}:=\left\{x \in \mathbb{R}^{n}:|x| \leq \rho\right\}$. If $V(P)$ satisfies $\operatorname{SPL}(1,0)$ then it also satisfies the condition $\widetilde{\mathrm{PL}}\left(K_{1}, K_{2}\right)$ defined in Franken and Meise [7], 2.6(c). Hence it follows from [7], 2.9, 2.11, and 2.13 that $P(D): C^{\infty}(\stackrel{\circ}{K} 1) \rightarrow C^{\infty}(\stackrel{\circ}{K} 1)$ admits a continuous linear right inverse. By Meise, Taylor, and Vogt [11], 3.8, this implies the conclusion.

\section{Local conditions}

In this section we introduce the localization $(P)_{\theta}$ of a given polynomial $P$ at a point $\theta \in V(P)$, which is used in the next section to formulate one 
of the main results. To derive properties of $(P)_{\theta}$ from those of $P$, we also consider local Phragmén-Lindelöf conditions and investigate some of their properties.

Definition 3.1. Let $V$ be an algebraic variety in $\mathbb{C}^{n}$ and $\xi \in V \cap \mathbb{R}^{n}$.

(a) $V$ satisfies the condition $\mathrm{PL}_{\mathrm{loc}}(\xi)$ if there exist constants $r_{1}>r_{2}>0$ and $A>0$ such that each plurisubharmonic function $u$ on $V \cap\{z \in$ $\left.\mathbb{C}^{n}:|z-\xi|<r_{1}\right\}$ that satisfies $(\alpha)$ and $(\beta)$ also satisfies $(\gamma)$, where

( $\alpha) u(z) \leq 1, z \in V \cap\left\{z \in \mathbb{C}^{n}:|z-\xi|<r_{1}\right\}$,

(ß) $u(z) \leq 0, z \in V \cap \mathbb{R}^{n} \cap\left\{z \in \mathbb{C}^{n}:|z-\xi|<r_{1}\right\}$,

$(\gamma) u(z) \leq A|\operatorname{Im} z|, z \in V \cap\left\{z \in \mathbb{C}^{n}:|z-\xi|<r_{2}\right\}$.

(b) Let $\eta: \mathbb{C}^{n} \rightarrow[0, \infty[$ be a function which satisfies $\eta(z)=o(|z|)$ as $|z|$ tends to zero. Then we say that $V$ satisfies the condition $\operatorname{PL}_{\text {loc }}(\xi, \eta)$ if there exist constants $r_{1}>r_{2}>0$ and $A>0$ such that for every $\rho>0$ there is a constant $B_{\rho}>0$ such that each plurisubharmonic function $u$ on $V \cap\left\{z \in \mathbb{C}^{n}:|z-\xi|<r_{1}\right\}$ which satisfies $(\alpha)$ and $(\beta)$ also satisfies $(\gamma)$, where

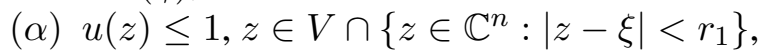

(ß) $u(z) \leq \rho|\operatorname{Im} z|, z \in V \cap\left\{z \in \mathbb{C}^{n}:|z-\xi|<r_{1}\right\}$,

$(\gamma) u(z) \leq A|\operatorname{Im} z|+B_{\rho} \eta(z-\xi), z \in V \cap\left\{z \in \mathbb{C}^{n}:|z-\xi|<r_{2}\right\}$.

Clearly, $\mathrm{PL}_{\mathrm{loc}}(\xi)$ implies $\mathrm{PL}_{\text {loc }}(\xi, \eta)$ for each function $\eta$ as in 3.1(b).

Remark 3.2. Classical estimates for the harmonic measure of the real line in the unit disk show that $\mathrm{PL}_{\text {loc }}(\xi)$ holds at $\xi \in V \cap \mathbb{R}^{n}$ whenever $V$ is a manifold in $\xi$ and $\operatorname{dim}_{\mathbb{R}} V \cap \mathbb{R}^{n}=\operatorname{dim}_{\mathbb{C}} V$ in $\xi$ (see, e.g., Meise, Taylor, and Vogt [11], Corollary 4.8).

From Meise and Taylor [9], Proposition 4.4, we recall the following result:

Proposition 3.3. If an algebraic variety $V$ in $\mathbb{C}^{n}$ satisfies (SPL), then it also satisfies $\mathrm{PL}_{\mathrm{loc}}(\xi)$ for each $\xi \in V \cap \mathbb{R}^{n}$.

The converse of Proposition 3.3 is false, since $V:=\left\{\left(z_{1}, z_{2}\right) \in \mathbb{C}^{2}\right.$ : $\left.z_{1}^{2}=z_{2}\right\}$ satisfies $\mathrm{PL}_{\text {loc }}(\xi)$ at each $\xi \in V \cap \mathbb{R}^{2}$ but it does not satisfy (SPL) by [9], Proposition 2.3 and Theorem 3.4.

Definition 3.4. Let $\theta \in \mathbb{C}^{n}$ and a function $P$ holomorphic in some neighborhood of $\theta$ be given. Then the lowest order homogeneous polynomial $(P)_{\theta}$ in the Taylor series expansion of $z \mapsto P(\theta+z)$ will be called the localization of $P$ in the point $\theta$.

In Chapter 7 of Whitney [17] several possibilities to introduce tangent varieties for analytic varieties are discussed. Here we only recall [17], 7.1G, the one which is defined in terms of limiting directions. 
Definition 3.5. Let $V$ be an analytic variety in $\mathbb{C}^{n}$ and $\zeta \in V$. The tangent cone $V_{\tan }(\zeta)$ in $\zeta$ is defined as the set of all $v \in \mathbb{C}^{n}$ such that for each $\varepsilon>0$ there exist $z \in V$ and $t \in \mathbb{C}$ satisfying $|z-\zeta|<\varepsilon$ and $|t(z-\zeta)-v|<\varepsilon$.

Remark 3.6. In Whitney [17], Theorem 7.4D, it is shown that $V_{\tan }(\zeta)$ can also be described as the common zero set of all localizations $(f)_{\zeta}$ of functions $f$ which are holomorphic in some neighborhood $U_{f}$ of $\zeta$ and vanish on $U_{f} \cap V$. In particular, $V_{\tan }(\zeta)$ is a homogeneous algebraic variety.

In Sect. 5 below we need to know a result (Corollary 3.8) which can be easily derived from the following theorem.

Theorem 3.7. If an algebraic variety $V$ in $\mathbb{C}^{n}$ satisfies the condition $\mathrm{PL}_{\mathrm{loc}}$ $(\xi, \eta)$ at $\xi \in V \cap \mathbb{R}^{n}$ for some function $\eta$ on $\mathbb{C}^{n}$ satisfying $\eta(z)=o(|z|)$, then the variety $V_{\tan }(\xi)$ satisfies $\mathrm{PL}_{\mathrm{loc}}(0)$ and $(\mathrm{SPL})$.

We do not include a proof of Theorem 3.7, because it is similar to published proofs of other results which show that certain Phragmén-Lindelöf conditions holding on an algebraic variety carry over to limit varieties. The first result of this type is due to Hörmander [8], who showed that the global Phragmén-Lindelöf condition that characterizes surjectivity of constant coefficient partial differential operators $P(D)$ on real analytic functions not only carries over to the cone of limiting directions of $V(P)$ at infinity, but is equivalent to the condition holding on this cone. In Meise, Taylor, and Vogt [15], Theorem 4.1, it was shown that the global Phragmén-Lindelöf condition $\operatorname{PL}\left(\mathbb{R}^{n}, \omega\right)$ carries over to the cone of limiting directions at infinity, while Braun [2], Theorem 4.1, proves the corresponding result for a local Phragmén-Lindelöf condition which is quite similar to $\mathrm{PL}_{\text {loc. }}$. Note that by Meise, Taylor, and Vogt [15], Theorem 3.3, $V_{\tan }(\xi)$ satisfies $\mathrm{PL}_{\mathrm{loc}}(0)$ if and only if $V_{\tan }(\xi)$ satisfies $\operatorname{PL}\left(\mathbb{R}^{n}, \log \right)$, which is equivalent to $(\mathrm{SPL})$, as we have noted in Lemma 2.5 .

Corollary 3.8. For $P \in \mathbb{C}\left[z_{1}, \ldots, z_{n}\right]$ let $V(P)$ satisfy $\mathrm{PL}_{\mathrm{loc}}(\theta)$ at some $\theta \in V(P) \cap \mathbb{R}^{n}$. Then each irreducible factor of $(P)_{\theta}$ is a constant multiple of a polynomial with real coefficients.

Proof. If $V(P)$ satisfies $\mathrm{PL}_{\mathrm{loc}}(\theta)$ then

$$
V_{\tan }(\theta)=\left\{z \in \mathbb{C}^{n}:(P)_{\theta}(z)=0\right\}
$$

satisfies (SPL) by Theorem 3.7 and hence $\operatorname{PL}\left(\mathbb{R}^{n}, \log \right)$. Consequently, it follows from Meise, Taylor, and Vogt [13], Lemma 2, that every irreducible factor of $(P)_{\theta}$ has real coefficients, up to a complex constant.

Next we provide two lemmas which will be applied in the following section and in which localizations are used. 
Lemma 3.9. For $n \geq 2$ let $P_{m} \in \mathbb{C}\left[z_{1}, \ldots, z_{n}\right]$ be homogeneous of degree $m \geq 2$, let $\theta:=(0, \ldots, 0,1)$, and assume $P_{m}(\theta)=0$. Then there exist $\nu \in \mathbb{N}, \nu \leq m$, and polynomials $Q_{j} \in \mathbb{C}\left[z_{1}, \ldots, z_{n-1}\right]$, homogeneous of degree $j, \nu \leq j \leq m$, such that $P_{m}\left(z^{\prime}, z_{n}\right)=\sum_{j=\nu}^{m} z_{n}^{m-j} Q_{j}\left(z^{\prime}\right)$. Moreover, $\left(P_{m}\right)_{\theta}\left(z^{\prime}, z_{n}\right)=Q_{\nu}\left(z^{\prime}\right)$.

Proof. Applying Taylor's formula at $\theta, P_{m}(\theta)=0$ implies

$$
P_{m}\left(z^{\prime}, 1\right)=P_{m}\left(\theta+\left(z^{\prime}, 0\right)\right)=\sum_{j=\nu}^{m} Q_{j}\left(z^{\prime}\right)
$$

where $Q_{j} \in \mathbb{C}\left[z_{1}, \ldots, z_{n-1}\right]$ is zero or homogeneous of degree $j$ and where $Q_{\nu} \neq 0$. From $P_{m}(\theta)=0$ and $P_{m} \neq 0$ it follows that $1 \leq \nu \leq m$. Since $P_{m}$ is homogeneous, (3.1) implies for $z_{n} \neq 0$ :

$$
P_{m}\left(z^{\prime}, z_{n}\right)=z_{n}^{m} P_{m}\left(\frac{z^{\prime}}{z_{n}}, 1\right)=\sum_{j=\nu}^{m} z_{n}^{m-j} Q_{j}\left(z^{\prime}\right) .
$$

By continuity, this holds also when $z_{n}=0$. From (3.2) we get for $z=$ $\left(z^{\prime}, z_{n}\right)$

$$
\begin{aligned}
P_{m}(\theta+z) & =P_{m}\left(z^{\prime}, 1+z_{n}\right)=\sum_{j=\nu}^{m}\left(1+z_{n}\right)^{m-j} Q_{j}\left(z^{\prime}\right) \\
& =\sum_{j=\nu}^{m} \sum_{k=0}^{m-j}\left(\begin{array}{c}
m-j \\
k
\end{array}\right) z_{n}^{k} Q_{j}\left(z^{\prime}\right)
\end{aligned}
$$

and hence $\left(P_{m}\right)_{\theta}\left(z^{\prime}, z_{n}\right)=Q_{\nu}\left(z^{\prime}\right)$.

Lemma 3.10. Let $P_{m} \in \mathbb{C}\left[z_{1}, \ldots, z_{n}\right]$ be homogeneous of degree $m \geq 2$. If $\xi:=(1,0, \ldots, 0) \in V\left(P_{m}\right)$ and if the localization $\left(P_{m}\right)_{\xi}$ of $P_{m}$ at $\xi$ has degree $\nu$, then

$$
\begin{aligned}
& s:(\tau, z) \mapsto P_{m}\left(z_{1} \tau^{-\nu}, z_{2} \tau^{m-\nu}, \ldots, z_{n} \tau^{m-\nu}\right), \\
& (\tau, z) \in(\mathbb{C} \backslash\{0\}) \times \mathbb{C}^{n},
\end{aligned}
$$

extends to a polynomial $\widetilde{s} \in \mathbb{C}\left[\tau, z_{1}, \ldots, z_{n}\right]$ with $\widetilde{s}(0, z)=z_{1}^{m-\nu}\left(P_{m}\right)_{\xi}$ $\left(z_{2}, \ldots, z_{n}\right)$.

Proof. It is easy to check that for $z=\left(z_{1}, z^{\prime}\right)$

$$
P_{m}\left(z_{1}, z^{\prime}\right)=\sum_{j=\nu}^{m} z_{1}^{m-j} q_{j}\left(z^{\prime}\right)
$$


where the polynomials $q_{j}$ are either zero or homogeneous of degree $j$. By definition, $q_{\nu}=\left(P_{m}\right)_{\xi}$. From this representation we get

$$
s(\tau, z)=\sum_{j=\nu}^{m} z_{1}^{m-j} \tau^{-\nu(m-j)} \tau^{j(m-\nu)} q_{j}\left(z^{\prime}\right)=\sum_{j=\nu}^{m} \tau^{m(j-\nu)} z_{1}^{m-j} q_{j}\left(z^{\prime}\right) .
$$

This shows that $s$ is the restriction of a polynomial $\widetilde{s}$ to $(\mathbb{C} \backslash\{0\}) \times \mathbb{C}^{n}$ and that

$$
s(0, z)=z_{1}^{m-\nu} q_{\nu}\left(z^{\prime}\right)=z_{1}^{m-\nu}\left(P_{m}\right)_{\xi}\left(z^{\prime}\right) .
$$

\section{A necessary condition for (SPL)}

Let $P \in \mathbb{C}\left[z_{1}, \ldots, z_{n}\right]$ have real principal part $P_{m}$ of degree $m \geq 2$ and define the polynomial $S$ by $S\left(z_{1}, \ldots, z_{n+1}\right):=P\left(z_{1}, \ldots, z_{n}\right)-z_{n+1}$. In this section we use the concept of quasihomogeneity and a result of [4] to derive a necessary condition in terms of $P_{m}$ for $V(S)$ to have $\mathrm{PL}\left(\mathbb{R}^{n+1}, \log \right)$. This extends a result of Meise and Taylor [9] and explains some of their counterexamples. The precise result is stated in the following theorem.

Theorem 4.1. Let $P \in \mathbb{C}\left[z_{1}, \ldots, z_{n}\right]$ have degree $m \geq 2$ and principal part $P_{m} \in \mathbb{R}\left[z_{1}, \ldots, z_{n}\right]$. Define $S \in \mathbb{C}\left[z_{1}, \ldots, z_{n+1}\right]$ by $S\left(z^{\prime}, z_{n+1}\right):=$ $P\left(z^{\prime}\right)-z_{n+1}$. If $V(S)$ satisfies $\operatorname{PL}\left(\mathbb{R}^{n+1}, \log \right)$, then for each $\theta \in V\left(P_{m}\right) \cap$ $\mathbb{R}^{n},|\theta|=1$, the localization $\left(P_{m}\right)_{\theta}$ of $P_{m}$ in $\theta$ is square-free.

The proof of this theorem is prepared by several lemmas for which we need the following definition.

Definition 4.2. For $d=\left(d_{1}, \ldots, d_{n}\right) \neq(0, \ldots, 0)$ with $d_{j}$ non-negative and rational for $1 \leq j \leq n$, a given polynomial $P \in \mathbb{C}\left[z_{1}, \ldots, z_{n}\right]$ is said to be $d$-quasihomogeneous of degree $m \geq 0$ if

$$
P(z)=\sum_{\langle d, \alpha\rangle=m} a_{\alpha} z^{\alpha}, \quad z \in \mathbb{C}^{n},
$$

where $\langle d, \alpha\rangle=\sum_{j=1}^{n} d_{j} \alpha_{j}$ and where not all $a_{\alpha}$ vanish. The zero polynomial is considered to be $d$-quasihomogeneous of degree $-\infty$.

For an arbitrary polynomial $Q \in \mathbb{C}\left[z_{1}, \ldots, z_{n}\right]$ the $d$-quasihomogeneous principal part of $Q$ is defined as the highest degree $d$-quasihomogeneous part of $Q$.

The concept of quasihomogeneity is relevant for our considerations because of the following lemma which we recall from [4], Lemma 3.2. 
Lemma 4.3. Let $P \in \mathbb{C}\left[z_{1}, \ldots, z_{n}\right]$ be d-quasihomogeneous of degree $m>$ 0 and let $Q \in \mathbb{C}\left[z_{1}, \ldots, z_{n}\right]$ be a sum of $d$-quasihomogeneous polynomials of degrees less than $\mathrm{m}$. Assume further that the following conditions are fulfilled:

(1) $d_{1}<d_{j}$ for $2 \leq j \leq n$,

(2) there exists $\zeta=\left(\zeta_{1}, \zeta^{\prime \prime}\right) \in V(P)$ with $\zeta_{1} \notin \mathbb{R}, \zeta^{\prime \prime} \in \mathbb{R}^{n-1}$, and $\zeta^{\prime \prime} \neq 0$,

(3) the polynomial $\lambda \mapsto P\left(\lambda, \zeta^{\prime \prime}\right)$ does not vanish identically.

If $V(P+Q)$ satisfies $\mathrm{PL}\left(\mathbb{R}^{n}, \omega\right)$ for some weight function $\omega$ and $D:=$ $\max \left\{d_{j}: \zeta_{j} \neq 0\right\}$, then $\omega$ satisfies $t^{d_{1} / D}=O(\omega(t))$ as t tends to infinity.

Lemma 4.4. For $n \geq 3$ let $P \in \mathbb{C}\left[z_{1}, \ldots, z_{n}\right]$ be a polynomial of degree $m \geq 2$ with principal part $P_{m}$ which satisfies $P_{m}(\theta)=0$ for $\theta=$ $(0, \ldots, 0,1) \in \mathbb{R}^{n}$. Assume that there exist $k \geq 2, g \geq 1$, and $Q, R \in$ $\mathbb{C}\left[z_{1}, \ldots, z_{n-1}\right]$ such that the following conditions are satisfied:

(a) $\left(P_{m}\right)_{\theta}\left(z^{\prime}, z_{n}\right)=Q\left(z^{\prime}\right)^{k} R\left(z^{\prime}\right)$ for $z=\left(z^{\prime}, z_{n}\right) \in \mathbb{C}^{n}$,

(b) $Q\left(z^{\prime}\right)=\sum_{|\alpha|=g} a_{\alpha}\left(z^{\prime}\right)^{\alpha}$ where $a_{1, g-1,0, \ldots, 0} \neq 0$ and $a_{\alpha}=0$ for all $\alpha \in \mathbb{N}_{0}^{n-1}$ satisfying $\alpha_{1}=0$ and $\alpha_{2} \geq g-1$.

Then the zero variety of the polynomial $S \in \mathbb{C}\left[z_{1}, \ldots, z_{n+1}\right]$ defined by

$$
S\left(z_{1}, \ldots, z_{n+1}\right)=P\left(z_{1}, \ldots, z_{n}\right)-z_{n+1}
$$

does not satisfy $\mathrm{PL}\left(\mathbb{R}^{n+1}, \log \right)$.

Proof. For $P_{m}$ and $\theta$ choose $\nu \in \mathbb{N}, 1 \leq \nu \leq m$, and polynomials $Q_{j} \in$ $\mathbb{C}\left[z_{1}, \ldots, z_{n-1}\right], \nu \leq j \leq m$, according to Lemma 3.9. Because of (a) we get from Lemma 3.9 that $S$ is of the following form:

$$
\begin{aligned}
S\left(z^{\prime}, z_{n}, z_{n+1}\right)= & z_{n}^{m-\nu} Q\left(z^{\prime}\right)^{k} R\left(z^{\prime}\right) \\
& +\sum_{j=\nu+1}^{m} z_{n}^{m-j} Q_{j}\left(z^{\prime}\right)+\widetilde{P}\left(z^{\prime}, z_{n}\right)-z_{n+1},
\end{aligned}
$$

where $\operatorname{deg} \widetilde{P}<m$. In order to apply Lemma 4.3, we have to find an appropriate quasihomogeneity $d$ such that the $d$-quasihomogeneous principal part of $S$ admits roots whose imaginary parts have a special form. The definition of the first $n$ weights is

$$
\begin{gathered}
d_{1}:=1, \quad d_{2}:=1+\frac{1}{m^{2}}, \quad d_{3}:=\ldots:=d_{n-1}:=1+\frac{1}{3 m^{2}}, \\
d_{n}:=1+\frac{1}{m}+\frac{1}{m^{2}}, \quad d^{\prime}:=\left(d_{1}, \ldots, d_{n-1}\right) .
\end{gathered}
$$


Denote the $d^{\prime}$-quasihomogeneous principal part of $R$ by $B$ and let $A=$ $d^{\prime}-\operatorname{deg} B$. Note that $A$ is not smaller than the classical degree of $B$, i.e.,

$$
A \geq \nu-k g \text {. }
$$

Now define

$$
d_{n+1}:=(m-\nu) d_{n}+k\left(g+\frac{g-1}{m^{2}}\right)+A, \quad d:=\left(d^{\prime}, d_{n}, d_{n+1}\right) .
$$

Note that (4.1) implies $d_{n+1} \geq m$. We claim:

(*) The $d^{\prime}$-quasihomogeneous principal part of $Q$ is $a_{1, g-1,0, \ldots, 0} z_{1} z_{2}^{g-1}$, whose $d^{\prime}$-degree is $g+(g-1) m^{-2}$.

To prove this claim, let $\alpha \neq(1, g-1,0, \ldots, 0)$ with $a_{\alpha} \neq 0$ be given. There are three cases:

Case $\alpha_{1}=0$ Then $\alpha_{2} \leq g-2$ because of (b). Since $d_{2}>d_{j}$ for $2<j<n$, we have the following estimate for the $d^{\prime}$-degree of $z^{\alpha}$ :

$$
\begin{aligned}
\sum_{j=2}^{n-1} \alpha_{j} d_{j} & \leq(g-2) d_{2}+2 d_{3}=g+\frac{g-2}{m^{2}}+\frac{2}{3 m^{2}} \\
& =g+\frac{g-4 / 3}{m^{2}}<g+\frac{g-1}{m^{2}} .
\end{aligned}
$$

Case $\alpha_{1}=1$ The case $\alpha_{2}=g-1$ being excluded, we have again $\alpha_{2} \leq$ $g-2$. Since $d_{2}>d_{j}>d_{1}$ for $2<j<n$, the $d^{\prime}$-degree of $z^{\alpha}$ is clearly smaller than that of $z_{1} z_{2}^{g-1}$.

Case $\alpha_{1} \geq 2$ The $d^{\prime}$-degree of $z^{\alpha}$ is

$$
\alpha_{1} d_{1}+\sum_{j=2}^{n-1} \alpha_{j} d_{j} \leq 2 d_{1}+(g-2) d_{2}=g+\frac{g-2}{m^{2}}<g+\frac{g-1}{m^{2}} .
$$

This proves $(*)$. The next claim is:

$(* *)$ The $d$-quasihomogeneous principal part of $S$ is

$$
S_{q}\left(z_{1}, \ldots, z_{n+1}\right):=z_{n}^{m-\nu} a_{1, g-1,0, \ldots, 0} z_{1}^{k} z_{2}^{k(g-1)} B\left(z^{\prime}\right)-z_{n+1} .
$$

To prove (**), note first that because of (4.2) the polynomial $S_{q}$ is $d$ quasihomogeneous of $d$-degree $d_{n+1}$. Furthermore, since $d_{j} \leq d_{n}$ for $1 \leq j \leq n$,

$$
d-\operatorname{deg} \widetilde{P} \leq(m-1) d_{n}=m-1+(m-1) \frac{m+1}{m^{2}}=m-\frac{1}{m^{2}}<d_{n+1} .
$$


To show that $z_{n}^{m-j} Q_{j}\left(z^{\prime}\right), \nu<j \leq m$, does not belong to the $d$-quasihomogeneous principal part of $S$, consider:

$$
\begin{aligned}
d_{n+1}-d-\operatorname{deg} z_{n}^{m-j} Q_{j}\left(z^{\prime}\right) \geq & (m-\nu) d_{n}+k\left(g+\frac{g-1}{m^{2}}\right) \\
& +A-(m-j) d_{n}-j d_{2} \\
\geq & (j-\nu) d_{n}+\nu-j d_{2} \\
= & \frac{j-\nu}{m}-\frac{\nu}{m^{2}} \geq \frac{1}{m}-\frac{m-1}{m^{2}}=\frac{1}{m^{2}} .
\end{aligned}
$$

So $(* *)$ is shown. Choose $\zeta^{\prime} \in \mathbb{R}^{n-1} \backslash\{0\}$ such that $p: \lambda \mapsto S_{q}\left(\lambda, \zeta^{\prime}, 0\right)$, $\lambda \in \mathbb{C}$, is a polynomial of degree at least $k \geq 2$. Fix now a value $b \in \mathbb{R}$ that $\left.p\right|_{\mathbb{R}}$ obtains at most once, let $\zeta_{1}$ be a non-real solution of $p\left(\zeta_{1}\right)=b$, and set $\zeta^{\prime \prime}=\left(\zeta^{\prime}, b\right)$. Then the claim follows from Lemma 4.3.

Lemma 4.5. For $n \geq 2$ let $Q \in \mathbb{R}\left[z_{1}, \ldots, z_{n}\right]$ be square-free and homogeneous of degree $g \geq 1$. Then there exists a real linear change of coordinates such that in the new coordinates $Q(z)=\sum_{|\alpha|=g} a_{\alpha} z^{\alpha}$, where $a_{\alpha} \neq 0$ for $\alpha=(g-1,1,0, \ldots, 0)$ and $a_{\alpha}=0$ whenever $\alpha_{1} \geq g-1$ and $\alpha_{2}=0$.

Proof. Since $Q$ is square-free, we can find $b_{1} \in \mathbb{R}^{n}$ satisfying $Q\left(b_{1}\right)=0$ and $\operatorname{grad} Q\left(b_{1}\right) \neq 0$. Next choose $b_{2} \in \mathbb{R}^{n}$ such that $\left\langle b_{2}, \operatorname{grad} Q\left(b_{1}\right)\right\rangle \neq 0$. Then Euler's rule, $\langle x, \operatorname{grad} Q(x)\rangle=g Q(x)$, implies that $b_{1}$ and $b_{2}$ are linearly independent. By our choice of $b_{1}$ and $b_{2}$, the function $g: \mathbb{R} \rightarrow \mathbb{R}$, $g(\zeta):=Q\left(b_{1}+\zeta b_{2}\right)$ satisfies

$$
g(0)=Q\left(b_{1}\right)=0 \quad \text { and } \quad g^{\prime}(0)=\left\langle\operatorname{grad} Q\left(b_{1}\right), b_{2}\right\rangle \neq 0 .
$$

Next choose $b_{3}, \ldots, b_{n}$ such that $\left\{b_{1}, \ldots, b_{n}\right\}$ is a basis of $\mathbb{R}^{n}$ and consider the following expansion

$$
Q\left(\sum_{k=1}^{n} \zeta_{k} b_{k}\right)=\sum_{j=0}^{g} q_{j}\left(\zeta_{2}, \ldots, \zeta_{n}\right) \zeta_{1}^{g-j}
$$

where the polynomials $q_{j}$ are homogeneous of degree $j$. In particular, (4.4) implies

$$
g(\zeta)=Q\left(b_{1}+\zeta b_{2}\right)=\sum_{j=0}^{g} q_{j}(\zeta, 0, \ldots, 0)=\sum_{j=0}^{g} q_{j}(1,0, \ldots, 0) \zeta^{j} .
$$

By (4.3) and the homogeneity properties of $q_{0}$, we conclude from this $q_{0}=0$ and $q_{1}(1,0, \ldots, 0) \neq 0$. Now choose $a \in \mathbb{R}^{n-1}$ such that $q_{1}(\xi)=\langle\xi, a\rangle$ 
and note that $a \neq 0$. Hence there is $M \in \mathrm{GL}(n-1, \mathbb{R})$ such that $M(a)=$ $(1,0, \ldots, 0)$. Define

$$
p\left(\sum_{j=1}^{n} \zeta_{j} b_{j}\right):=Q\left(\zeta_{1} b_{1}+\sum_{i=2}^{n}\left(\sum_{j=2}^{n} M_{j-1, i-1} \zeta_{j}\right) b_{i}\right) .
$$

Then the linear term of this polynomial is

$$
\begin{aligned}
q_{1}\left(\left(\sum_{j=2}^{n} M_{j-1, i-1} \zeta_{j}\right)_{i=2}^{n}\right) & =\left\langle M^{t}\left(\zeta_{2}, \ldots, \zeta_{n}\right), a\right\rangle \\
& =\left\langle\left(\zeta_{2}, \ldots, \zeta_{n}\right), M a\right\rangle=\zeta_{2} .
\end{aligned}
$$

Hence $p$ has the desired form.

Proof of Theorem 4.1. Note first that the principal part $S_{m}$ of $S$ is the polynomial $P_{m}$, regarded as an element of $\mathbb{R}\left[z_{1}, \ldots, z_{n+1}\right]$. Since $V(S)$ satisfies $\operatorname{PL}\left(\mathbb{R}^{n+1}, \log \right)$, it follows from Meise, Taylor, and Vogt [15], Theorem 4.1, that $V\left(S_{m}\right)=V\left(P_{m}\right) \times \mathbb{C}$ has the same property. Hence $V\left(P_{m}\right)$ satisfies $\mathrm{PL}\left(\mathbb{R}^{n}, \log \right)$ and consequently (SPL), by Proposition 2.5(b). Assume there exists $\theta \in V\left(P_{m}\right) \cap \mathbb{R}^{n},|\theta|=1$, such that $\left(P_{m}\right)_{\theta}$ is not square-free. After a suitable real linear change of variables we may assume $\theta=(0, \ldots, 0,1)$. By Lemma 3.9 and our assumption there exist $Q, R \in \mathbb{C}\left[z_{1}, \ldots, z_{n-1}\right], Q$ square-free, and $k \geq 2$ such that $\operatorname{deg} Q \geq 1, R \neq 0$, and

$$
\left(P_{m}\right)_{\theta}\left(z^{\prime}, z_{n}\right)=Q\left(z^{\prime}\right)^{k} R\left(z^{\prime}\right) .
$$

Since $P_{m}$ has real coefficients, so does $\left(P_{m}\right)_{\theta}$. As $V\left(P_{m}\right)$ satisfies (SPL), it follows from Corollary 3.8 that it is no restriction to assume that $Q$ and $R$ have real coefficients. If $n \geq 3$, then Lemma 4.5 shows that there exists a change of variables in the $z^{\prime}$ variables such that $Q$ has the special form given in condition (b) of Lemma 4.4. Hence Lemma 4.4 implies that $V(S)$ does not satisfy $\mathrm{PL}\left(\mathbb{R}^{n+1}, \log \right)$ in contradiction to the hypothesis. This proves Theorem 4.1 for $n \geq 3$.

If $n=2$ then it follows from Lemma 3.9 that

$$
P_{m}\left(z_{1}, z_{2}\right)=z_{2}^{m-\nu}\left(P_{m}\right)_{\theta}\left(z_{1}\right)+\sum_{j=\nu+1}^{m} z_{2}^{m-j} Q_{j}\left(z_{1}\right)
$$

for some $\nu \in \mathbb{N}$ and suitable polynomials $Q_{j}$ which are homogeneous of degree $j$. The assumption on $\left(P_{m}\right)_{\theta}$ implies $\nu \geq 2$. Hence

$$
S\left(z_{1}, z_{2}, z_{3}\right)=\sum_{j=\nu}^{m} a_{j} z_{2}^{m-j} z_{1}^{j}+\sum_{k=0}^{m-1} \sum_{|\alpha|=k} a_{\alpha} z_{1}^{\alpha_{1}} z_{2}^{\alpha_{2}}-z_{3},
$$


where $a_{\nu} \in \mathbb{R} \backslash\{0\}$. To apply Lemma 4.3 again, let

$$
d_{1}:=1, d_{2}:=\frac{m+1}{m} \text {, and } d_{3}:=d_{2}(m-\nu)+\nu .
$$

Then it is easy to check that the $d$-quasihomogeneous principal part of $S$ is the polynomial $Q\left(z_{1}, z_{2}, z_{3}\right):=a_{\nu} z_{2}^{m-\nu} z_{1}^{\nu}-z_{3}$. Now let $\zeta_{2}:=1$, $\zeta_{3}=-\operatorname{sign} a_{\nu}$, and note that $\nu \geq 2$. Hence there exists $\zeta_{1} \in \mathbb{C} \backslash \mathbb{R}$ such that $Q\left(\zeta_{1}, \zeta_{2}, \zeta_{3}\right)=0$. Consequently, Lemma 4.3 implies that $V(S)$ does not satisfy $\mathrm{PL}\left(\mathbb{R}^{3}, \log \right)$, in contradiction to the hypothesis. This proves Theorem 4.1 also if $n=2$.

Theorem 4.1 extends Meise and Taylor [9], Lemma 2.2, as the following corollary shows.

Corollary 4.6. Let $P \in \mathbb{C}\left[z_{1}, \ldots, z_{n}\right]$ have degree $m \geq 2$ and principal part $P_{m} \in \mathbb{R}\left[z_{1}, \ldots, z_{n}\right]$. Define $S \in \mathbb{C}\left[z_{1}, \ldots, z_{n+1}\right]$ by $S\left(z^{\prime}, z_{n+1}\right):=$ $P\left(z^{\prime}\right)-z_{n+1}$. If $V(S)$ satisfies $\mathrm{PL}\left(\mathbb{R}^{n+1}, \log \right)$, then $P_{m}$ is square-free.

Proof. As in the proof of Theorem 4.1 it follows that $V\left(P_{m}\right)$ satisfies $\mathrm{PL}\left(\mathbb{R}^{n}, \log \right)$. If we assume that $P_{m}$ is not square-free then there exist $Q, R \in \mathbb{C}\left[z_{1}, \ldots, z_{n}\right]$ and $k \geq 2$ such that $P_{m}=Q^{k} R$, where $\operatorname{deg} Q \geq 1$. Since $V\left(P_{m}\right)$ has $\operatorname{PL}\left(\mathbb{R}^{n}, \log \right)$, also $V(Q)$ has this property by Meise, Taylor, and Vogt [15], Proposition 2.6, and hence the real zero set of $Q$ has dimension $n-1$ by [15], Theorem 3.13. In particular there exists $\theta \in V(Q) \cap \mathbb{R}^{n},|\theta|=1$. Now it is easy to check that

$$
\left(P_{m}\right)_{\theta}=\left(Q_{\theta}\right)^{k} R_{\theta} .
$$

Hence Theorem 4.1 implies that $V(S)$ does not satisfy $\operatorname{PL}\left(\mathbb{R}^{n+1}, \log \right)$ in contradiction to the hypothesis.

\section{A characterization in dimension 3}

In this section we show that the necessary condition which we derived in Theorem 4.1 leads to a characterization of those homogeneous polynomials $P_{m}$ in three variables for which $V_{+}\left(P_{m}\right)$ and $V_{-}\left(P_{m}\right)$ have (SPL). The precise result is the following theorem which implies Theorem 1.1.

Theorem 5.1. Let $P_{m} \in \mathbb{C}\left[z_{1}, z_{2}, z_{3}\right]$ be homogeneous of degree $m \geq 2$ and define $P \in \mathbb{C}\left[z_{1}, \ldots, z_{4}\right]$ by $P\left(z_{1}, \ldots, z_{4}\right):=P_{m}\left(z_{1}, z_{2}, z_{3}\right)-z_{4}$. Then the following conditions are equivalent:

(1) $V_{+}\left(P_{m}\right)$ and $V_{-}\left(P_{m}\right)$ satisfy (SPL),

(2) $V(P)$ satisfies $(\mathrm{SPL})$,

(3) $V(P)$ satisfies $\mathrm{PL}\left(\mathbb{R}^{4}, \log \right)$, 
(4) $V\left(P_{m}\right)$ satisfies (SPL), $P_{m}$ has real coefficients, and for each $\theta \in$ $V\left(P_{m}\right) \cap \mathbb{R}^{3},|\theta|=1$, the localization of $P_{m}$ at $\theta$ is square-free.

The theorem is proved by showing that $V_{+}\left(P_{m}\right)$ and $V_{-}\left(P_{m}\right)$ inherit (SPL) from two simpler varieties. One of them is of course $V\left(P_{m}\right)$, the cone of limiting directions. The hypotheses imply that $V\left(P_{m}\right)$ is locally hyperbolic near $V\left(P_{m}\right) \cap \mathbb{R}^{3} \backslash\{0\}$. The a priori estimate of Theorem 5.2 allows us to restrict our attention to cones spanned by neighborhoods of points $\xi \in V\left(P_{m}\right)$. Assume $\xi=(1,0,0)$ and denote the vanishing order of $P_{m}$ in $\xi$ by $\nu$. In Lemma 5.6 we show that inside the above mentioned cone, but outside a tubular neighborhood $U$ of $\mathbb{R} \cdot \xi$, the two varieties $V(S)$ and $V\left(P_{m}\right)$ are so close that the standard method for proving Phragmén-Lindelöf conditions for hyperbolic polynomials, namely taking maxima over the fibers of a suitable projection, works also for $V(S)$. Inside $U$, however, $V(S)$ is still a manifold, while $V\left(P_{m}\right)$ may be singular in $\xi$. Here, we compare $V(S)$ to another manifold $V\left(Q_{0}-1\right)$, where $Q_{0}\left(z_{2}, z_{3}\right):=\left(P_{m}\right)_{\xi}(z)$. We show in Lemma 5.10 that coordinate patches for $V\left(Q_{0}-1\right)$ induce coordinate patches for $V(S) \cap U$ and use standard estimates on these patches. At first we recall a result from [5].

Theorem 5.2. Let $P_{m} \in \mathbb{R}\left[z_{1}, \ldots, z_{n}\right]$ be homogeneous of degree $m$ and let $Q \in \mathbb{R}\left[z_{1}, \ldots, z_{n}\right]$ be of degree less than $m$. If $P_{m}$ is square-free and if $V\left(P_{m}\right)$ satisfies $\mathrm{PL}\left(\mathbb{R}^{n}, \log \right)$ then there exist $A_{0} \geq 1, B_{0} \geq 0$ such that each $u \in \operatorname{PSH}\left(V\left(P_{m}+Q\right)\right)$ which satisfies the conditions $(\alpha)$ and $(\beta)$ of 2.2(a) also satisfies $u(z) \leq A_{0}|z|+B_{0}, z \in V\left(P_{m}+Q\right)$.

Proof. Since $V\left(P_{m}\right)$ has $\mathrm{PL}\left(\mathbb{R}^{n}, \log \right)$, it follows from Meise, Taylor, and Vogt [15], Theorem 3.13, that each irreducible factor of $P_{m}$ has an $(n-1)$ dimensional set of real zeros. Therefore, the present theorem follows from [5], Theorem 1.1.

If $P_{m}$ satisfies the hypotheses in 5.1(4) then Theorem 5.2 and Lemma 2.5 imply that on $V_{ \pm}\left(P_{m}\right)$ the estimate needed for (SPL) holds whenever $z \in V_{ \pm}\left(P_{m}\right)$ satisfies $|z| \leq \lambda|\operatorname{Im} z|$ for some constant $\lambda>0$. Hence we only have to consider those points in $V_{ \pm}\left(P_{m}\right)$ for which $|\operatorname{Im} z| /|z|$ is small. This will be done in cones around real lines in $V\left(P_{m}\right)$. To handle these we need several lemmas. Furthermore, we will use the following notation.

Notation. For $w \in \mathbb{C}^{n}$ and $r=\left(r_{1}, \ldots, r_{n}\right), r_{j}>0,1 \leq j \leq n$, we denote the polydisk with center $w$ and polyradius $r$ by $B(w ; r)$ or $B\left(w ; r_{1}, \ldots, r_{n}\right)$. For $\rho>0$ we will use the abbreviation $B(w ; \rho)=B(w ; \rho, \ldots, \rho)$.

Lemma 5.3. Let $P_{m} \in \mathbb{R}\left[z_{1}, z_{2}, z_{3}\right]$ be homogeneous of degree $m \geq 2$ and assume that $V\left(P_{m}\right)$ has $\mathrm{PL}\left(\mathbb{R}^{3}, \log \right)$. Then for each $\xi \in V\left(P_{m}\right) \cap \mathbb{R}^{3}$, $|\xi|=1$, there exist a real linear change of variables, $\nu \in \mathbb{N}, \eta>0, \sigma>0$, 
and holomorphic functions $\beta_{j}: B((1,0) ; \eta, \eta) \rightarrow \mathbb{C}, 1 \leq j \leq \nu$, such that in the new coordinates the following holds:

(1) $\xi=(1,0,0)$, and $(0,0,1)$ is non-characteristic for $P_{m}$,

(2) $V\left(P_{m}\right) \cap B(\xi ; \eta, \eta, \sigma)=\bigcup_{j=1}^{\nu}\left\{\left(z_{1}, z_{2}, \beta_{j}\left(z_{1}, z_{2}\right)\right):\left(z_{1}, z_{2}\right) \in B\right.$ $((1,0) ; \eta, \eta)\}$

(3) $\beta_{j}\left(z_{1}, z_{2}\right)$ is real for real $z_{1}, z_{2}, 1 \leq j \leq \nu$.

Proof. It is easy to check that (1) can be achieved by a real linear change of variables. Since $P_{m}$ is real and $(0,0,1)$ is non-characteristic for $P_{m}$, up to a real constant we have

$$
P_{m}\left(z_{1}, z_{2}, z_{3}\right)=\prod_{j=1}^{m}\left(z_{3}-\beta_{j}\left(z_{1}, z_{2}\right)\right), \quad\left(z_{1}, z_{2}, z_{3}\right) \in \mathbb{C}^{3},
$$

where $\beta_{1}\left(z_{1}, z_{2}\right), \ldots, \beta_{m}\left(z_{1}, z_{2}\right)$ denote the (not necessarily distinct) $m$ roots of $z_{3} \mapsto P_{m}\left(z_{1}, z_{2}, z_{3}\right)$ for fixed $\left(z_{1}, z_{2}\right) \in \mathbb{C}^{2}$. By hypothesis $P_{m}(1,0,0)$ $=0$, therefore there exists $\nu$ with $1 \leq \nu \leq m$ such that (without restriction)

$\beta_{j}(1,0)=0$ for $1 \leq j \leq \nu$ and $\beta_{j}(1,0)=a_{j} \neq 0$ for $\nu+1 \leq j \leq m$.

Since $V\left(P_{m}\right)$ satisfies $\operatorname{PL}\left(\mathbb{R}^{3}, \log \right)$ it follows from Braun [1], Corollary 12, that there are $0<\eta, \sigma \leq 1$, and holomorphic functions $\beta_{j}: B((1,0) ; \eta, \eta)$ $\rightarrow \mathbb{C}, 1 \leq j \leq \nu$, such that

$$
\begin{aligned}
& V\left(P_{m}\right) \cap B(\xi ; \eta, \eta, \sigma) \\
& \quad=\bigcup_{j=1}^{\nu}\left\{\left(z_{1}, z_{2}, \beta_{j}\left(z_{1}, z_{2}\right)\right):\left(z_{1}, z_{2}\right) \in B((1,0) ; \eta, \eta)\right\} .
\end{aligned}
$$

Moreover, $\beta_{j}\left(z_{1}, z_{2}\right)$ is real when $\left(z_{1}, z_{2}\right)$ is real for $1 \leq j \leq \nu$. Hence (2) and (3) hold.

The easy proof of the following lemma is left to the reader.

Lemma 5.4. Let $\nu \in \mathbb{N}$ and distinct numbers $b_{1}, \ldots, b_{\nu} \in \mathbb{R} \backslash\{0\}$ be given and let $Q_{0}\left(z_{2}, z_{3}\right):=\prod_{j=1}^{\nu}\left(z_{3}-b_{j} z_{2}\right)$. Then there exist $\rho_{0} \geq 1$ and $D_{0}>0$ such that for each $\mu \in \mathbb{C},|\mu| \leq 2$ and each $\left(w_{2}, w_{3}\right) \in \mathbb{C}^{2}$ satisfying $Q_{0}\left(w_{2}, w_{3}\right)=\mu$ and $\left|w_{2}\right| \geq \rho_{0}$ there exists a unique $l, 1 \leq l \leq \nu$, such that

$$
\left|w_{3}-b_{l} w_{2}\right| \leq \frac{D_{0}}{\left|w_{2}\right|^{\nu-1}}
$$


Lemma 5.5. For $p \in \mathbb{N}$ there exists $C \geq 1$ such that for any choice of $A$ and $a \in \mathbb{C}^{p}$ satisfying $\left|a_{j}\right|<\left|A_{j}\right| / 2$ for $1 \leq j \leq p$, the following estimate holds:

$$
\left|\prod_{j=1}^{p}\left(A_{j}+a_{j}\right)-\prod_{j=1}^{p} A_{j}\right| \leq C \max _{1 \leq k \leq p}\left(\left|a_{k}\right| \prod_{j \neq k} A_{j}\right) .
$$

Proof. Writing the difference as a telescoping sum, the estimate is obtained easily.

Lemma 5.6. Let $P_{m} \in \mathbb{R}\left[z_{1}, z_{2}, z_{3}\right]$ be homogeneous of degree $m \geq 2$ and satisfy condition 5.1(4). Assume that $\xi=(1,0,0) \in V\left(P_{m}\right)$ and that the conclusions of Lemma 5.3 hold. Then there exist $\rho_{0} \geq 1, r, s>0, D>0$, and $R_{1}>1$ such that for each $\geq R_{1}$ and $\left(z_{1}, z_{2}\right) \in \mathbb{C}^{2}$ satisfying $\left|z_{1}\right|<r t$ and $t^{1-m / \nu} \rho_{0}<\left|z_{2}\right|<r t$ the equation $P_{m}\left(z_{1}, z_{2}, z_{3}\right)= \pm 1$ has exactly $\nu$ distinct roots $z_{3}^{1}\left(z_{1}, z_{2}\right), \ldots, z_{3}^{\nu}\left(z_{1}, z_{2}\right)$ satisfying $\left|z_{3}^{j}\left(z_{1}, z_{2}\right)\right| \leq$ st and $\left|z_{3}^{j}\left(z_{1}, z_{2}\right)\right| \leq D\left|z_{2}\right|$. Moreover, each $z_{3}^{j}\left(z_{1}, z_{2}\right)$ is real for real $z_{1}, z_{2}$.

Proof. We will give the proof only for the case $P_{m}(z)=+1$, since the one for $P_{m}=-1$ is the same, up to obvious modifications. In the proof we will use the notation that was introduced in Lemma 5.3. Since $\left(P_{m}\right)_{\xi}$ is square-free, the real numbers

$$
b_{j}:=\frac{\partial \beta_{j}}{\partial z_{2}}(1,0), \quad 1 \leq j \leq \nu,
$$

are distinct. Since the functions $\beta_{j}, 1 \leq j \leq \nu$, are holomorphic, we have the following power series expansions

$$
\beta_{j}(1, \lambda)=b_{j} \lambda+\sum_{k=2}^{\infty} b_{j, k} \lambda^{k}, \quad|\lambda|<\eta, \quad 1 \leq j \leq \nu .
$$

Next define the holomorphic functions

$$
\begin{aligned}
& F, U: B(\xi ; \eta, \eta, \sigma) \rightarrow \mathbb{C}, \quad F(z)=\prod_{j=1}^{\nu}\left(z_{3}-\beta_{j}\left(z_{1}, z_{2}\right)\right), \\
& U(z):=\frac{P_{m}(z)}{F(z)}
\end{aligned}
$$

Since $(0,0,1)$ is non-characteristic for $P_{m}$, the equation $P_{m}\left(1,0, z_{3}\right)=$ 0 has $m$ roots, $\nu$ of which are zero. The other roots, $a_{\nu+1}, \ldots, a_{m}$, are all non-zero and satisfy $\left|a_{j}\right|>\sigma$ by 5.3(2). It is no restriction to assume $P_{m}\left(1,0, z_{3}\right)=z_{3}^{\nu} \prod_{j=\nu+1}^{m}\left(-a_{j}\right)$ and $\prod_{j=\nu+1}^{m}\left(-a_{j}\right)=1$, which implies

$$
U(1,0,0)=\prod_{j=\nu+1}^{m}\left(-a_{j}\right)=1 .
$$


Shrinking $\eta$ and $\sigma$ if necessary, we may assume $\left|a_{j}\right|>3 \sigma$ for $\nu+1 \leq j \leq m$. Next note that for $z \in B(\xi ; \eta, \eta, \sigma)$ we have

$$
\begin{aligned}
F\left(z_{1}, z_{2}, z_{3}\right) & =\prod_{j=1}^{\nu}\left(z_{3}-z_{1} \beta_{j}\left(1, \frac{z_{2}}{z_{1}}\right)\right) \\
& =\prod_{j=1}^{\nu}\left(z_{3}-z_{2} b_{j}-g_{j}\left(z_{1}, z_{2}\right)\right),
\end{aligned}
$$

where

$$
g_{j}\left(z_{1}, z_{2}\right)=z_{1} \sum_{k=2}^{\infty} b_{j, k}\left(\frac{z_{2}}{z_{1}}\right)^{k} .
$$

From this it follows that, shrinking $\eta>0$ again, there exists $M>0$ such that

$$
\left|g_{j}\left(z_{1}, z_{2}\right)\right| \leq M\left|z_{2}\right|^{2}, \quad\left(z_{1}, z_{2}\right) \in B((1,0) ; \eta, \eta), \quad 1 \leq j \leq \nu .
$$

Now define $Q_{0} \in \mathbb{R}\left[z_{2}, z_{3}\right]$ and $Q \in \mathbb{R}\left[z_{1}, z_{2}, z_{3}\right]$ by

$$
Q_{0}\left(z_{2}, z_{3}\right):=\prod_{j=1}^{\nu}\left(z_{3}-b_{j} z_{2}\right), \quad Q\left(z_{1}, z_{2}, z_{3}\right)=z_{1}^{m-\nu} Q_{0}\left(z_{2}, z_{3}\right) .
$$

To prove the lemma we will apply a scaling argument and Rouché's theorem. The basic observation for the scaling is that $P_{m}(z)=1$ is equivalent to $P_{m}(z / t)=t^{-m}$. If $z / t \in B(\xi ; \eta, \eta, \sigma)$, this equation is equivalent to $F(z / t)=t^{-m} / U(z / t)$. To get the desired information on the zeros of $P_{m}=1$ we approximate $F$ by $Q_{0}$ and $U$ by $z_{1}^{m-\nu}$. To do so, we need a number of estimates. To derive them, denote by $\rho_{0}$ and $D_{0}$ the numbers which exist for the polynomial $Q_{0}$ by Lemma 5.4. Furthermore, define

$$
\begin{aligned}
& \kappa:=\min \left\{\left|b_{j}-b_{k}\right|: 1 \leq j, k \leq \nu, j \neq k\right\}>0, \\
& M_{0}:=\max _{1 \leq j \leq \nu}\left|b_{j}\right| .
\end{aligned}
$$

Enlarging $\rho_{0}$ if necessary, we may assume $D_{0} / \rho_{0}^{\nu} \leq \min \left(\kappa / 2, M_{0},(\kappa / 2)^{\nu}\right.$ $\left.\left(4(\nu-1)\left(3 M_{0}\right)^{\nu-1}\right)^{-1}\right)$. Then fix $t>\left(2 \rho_{0} / \eta\right)^{\nu / m}$ and $z \in B(\xi ; \eta, \eta, \sigma)$ satisfying $\left|z_{2}\right|>\rho_{0} t^{-m / \nu}$. If $Q(z)=t^{-m}$ then the definition of $Q$ implies

$$
Q_{0}\left(t^{m / \nu} z_{2}, t^{m / \nu} z_{3}\right)=\frac{1}{z_{1}^{m-\nu}} .
$$

Provided that $\eta$ is small enough, we have

$$
\frac{1}{\left|z_{1}\right|^{m-\nu}} \leq\left(\frac{1}{1-\eta}\right)^{m-\nu} \leq 2
$$


Since $\left|t^{m / \nu} z_{2}\right|>\rho_{0}$, Lemma 5.4 implies the existence of a unique $l, 1 \leq$ $l \leq \nu$, satisfying $\left|t^{m / \nu} z_{3}-b_{l} t^{m / \nu} z_{2}\right| \leq D_{0} /\left|t^{m / \nu} z_{2}\right|^{\nu-1}$ and hence, using $\left|z_{2}\right|>\rho_{0} t^{-m / \nu}$

$$
\left|z_{3}-b_{l} z_{2}\right| \leq \frac{D_{0}}{t^{m}\left|z_{2}\right|^{\nu-1}} \leq \frac{D_{0}}{\rho_{0}^{\nu}}\left|z_{2}\right|
$$

By the definition of $\kappa$ and $M_{0}$ in (4.1), it follows from (5.5) and $\left|z_{2}\right|>$ $\rho_{0} t^{-m / \nu}$ that for $j \neq l$ and $1 \leq j \leq \nu$ we have the following estimates:

$$
\left|z_{3}-b_{j} z_{2}\right| \leq\left(\frac{D_{0}}{\rho_{0}^{\nu}}+2 M_{0}\right)\left|z_{2}\right| \leq 3 M_{0}\left|z_{2}\right|
$$

$$
\left|z_{3}-b_{j} z_{2}\right| \geq\left(\kappa-\frac{D_{0}}{\rho_{0}^{\nu}}\right)\left|z_{2}\right| \geq \frac{\kappa}{2}\left|z_{2}\right|
$$

Together with (5.5) they imply

$$
\begin{aligned}
\left|\frac{\partial Q_{0}}{\partial z_{3}}\left(z_{2}, z_{3}\right)\right| & =\left|\sum_{k=1}^{\nu} \prod_{j \neq k}\left(z_{3}-b_{j} z_{2}\right)\right| \\
& \geq\left(\frac{\kappa}{2}\left|z_{2}\right|\right)^{\nu-1}-(\nu-1)\left(3 M_{0}\right)^{\nu-2} \frac{D_{0}}{\rho_{0}^{\nu}}\left|z_{2}\right|^{\nu-1} \\
& \geq \frac{1}{2}\left(\frac{\kappa}{2}\left|z_{2}\right|\right)^{\nu-1} .
\end{aligned}
$$

By homogeneity there exists $K_{\nu} \in \mathbb{N}$ such that

$$
\left|\left(\frac{\partial}{\partial z_{3}}\right)^{p} Q_{0}\left(z_{2}, z_{3}\right)\right| \leq K_{\nu}\left|z_{2}\right|^{\nu-p}, p \geq 2 .
$$

Next choose $\delta>0$ so small that $\delta \leq \kappa / 4$ and $K_{\nu} \sum_{p=2}^{\nu} \delta^{p}<\frac{1}{4}\left(\frac{\kappa}{2}\right)^{\nu} \delta$. Since $Q(z)=t^{-m}$ it follows from (5.8) and (5.9) that for $\zeta \in \mathbb{C},|\zeta|=\delta\left|z_{2}\right|$, we have the following estimate 


$$
\begin{aligned}
\left|Q\left(z_{1}, z_{2}, b_{l} z_{2}+\zeta\right)-t^{-m}\right|= & \left|Q\left(z_{1}, z_{2}, b_{l} z_{2}+\zeta\right)-Q(z)\right| \\
= & \left|\sum_{p=1}^{\nu}\left(\frac{\partial}{\partial z_{3}}\right)^{p} Q_{0}\left(z_{2}, z_{3}\right) z_{1}^{m-\nu} \zeta^{p}\right| \\
\geq & \left(\frac{1}{2}\left(\frac{\kappa}{2}\left|z_{2}\right|\right)^{\nu-1} \delta\left|z_{2}\right|\right. \\
& \left.-K_{\nu} \sum_{p=2}^{\nu}\left|z_{2}\right|^{\nu-p} \delta^{p}\left|z_{2}\right|^{p}\right)\left|z_{1}\right|^{m-\nu} \\
\geq & \left(\frac{1}{4}\left(\frac{\kappa}{2}\right)^{\nu-1} \delta\left|z_{2}\right|^{\nu}\right)\left|z_{1}\right|^{m-\nu} \\
> & \frac{1}{8}\left(\frac{\kappa}{2}\right)^{\nu-1} \delta\left|z_{2}\right|^{\nu} .
\end{aligned}
$$

To derive an upper bound for $P_{m}\left(z_{1}, z_{2}, \cdot\right)-Q\left(z_{1}, z_{2}, \cdot\right)$ on the same circle, note that $Q=Q_{0} \cdot Q_{1}$, where $Q_{1}(z)=z_{1}^{m-\nu}$ and that

$$
P_{m}-Q=F U-Q_{0} Q_{1}=F\left(U-Q_{1}\right)+\left(F-Q_{0}\right) Q_{1}
$$

To estimate the terms on the right hand side, we use (5.3), (5.4), and Lemma 5.5 to get for $\lambda=b_{l} z_{2}+\zeta$ :

$$
\begin{aligned}
& \left|\left(\left(F-Q_{0}\right) Q_{1}\right)\left(z_{1}, z_{2}, \lambda\right)\right| \\
& \leq\left|\prod_{j=1}^{\nu}\left(\lambda-b_{j} z_{2}-g_{j}\left(z_{1}, z_{2}\right)\right)-\prod_{j=1}^{\nu}\left(\lambda-b_{j} z_{2}\right)\right|\left|z_{1}\right|^{m-\nu} \\
& \leq C \max _{1 \leq k \leq \nu}\left(\left|g_{j}\left(z_{1}, z_{2}\right)\right|\left|\prod_{j \neq k}\left(\lambda-b_{j} z_{2}\right)\right|\right)\left|z_{1}\right|^{m-\nu} \\
& \leq C M\left|z_{2}\right|^{2}\left(\left(2 M_{0}+\delta\right)\left|z_{2}\right|\right)^{\nu-1}\left|z_{1}\right|^{m-\nu} \leq L\left|z_{2}\right|^{\nu+1},
\end{aligned}
$$

where $L:=C M\left(2 M_{0}+\delta\right) 2^{m-\nu}$. Similarly we get

$$
\begin{aligned}
\left|F\left(z_{1}, z_{2}, \lambda\right)\right| & =\prod_{j=1}^{\nu}\left|\zeta+\left(b_{l}-b_{j}\right) z_{2}+g_{j}\left(z_{1}, z_{2}\right)\right| \\
& \leq\left(\delta\left|z_{2}\right|+M\left|z_{2}\right|^{2}\right)\left(\left(\delta+2 M_{0}\right)\left|z_{2}\right|\right)^{\nu-1} \\
& =\left(\delta+M\left|z_{2}\right|\right)\left(\delta+2 M_{0}\right)^{\nu-1}\left|z_{2}\right|^{\nu} .
\end{aligned}
$$


To estimate $U\left(z_{1}, z_{2}, \lambda\right)-z_{1}^{m-\nu}$, recall from the proof of Lemma 5.3 that for fixed $\left(z_{1}, z_{2}\right) \in B((1,0) ; \eta, \eta)$ we have, by the homogeneity of $P_{m}$,

$$
\begin{aligned}
U\left(z_{1}, z_{2}, \zeta\right) & =\prod_{j=\nu+1}^{m}\left(\zeta-\beta_{j}\left(z_{1}, z_{2}\right)\right) \\
& =\prod_{j=\nu+1}^{m}\left(\zeta-z_{1}\left(\beta_{j}\left(1, \frac{z_{2}}{z_{1}}\right)\right) .\right.
\end{aligned}
$$

Since $\beta_{j}(1,0)=a_{j}, \nu+1 \leq j \leq m$, and since the $\beta_{j}$ depend continuously on $z_{1}, z_{2}$, for each $\varepsilon>0$ there exists $\sigma(\varepsilon)>0$ such that $\left|\beta_{j}(1, z)-a_{j}\right| \leq \varepsilon$ for $|z| \leq \sigma(\varepsilon)$ and $\nu+1 \leq j \leq m$. Since $P_{m}$ is homogeneous and since $\prod_{j=\nu+1}^{m}\left(-a_{j}\right)=1$, we get from Lemma 5.5

$$
\begin{aligned}
& \left|U\left(z_{1}, z_{2}, \lambda\right)-z_{1}^{m-\nu}\right| \\
& =\left|\prod_{j=\nu+1}^{m}\left(\lambda-a_{j} z_{1}+\left(a_{j}-\beta_{j}\left(1, \frac{z_{2}}{z_{1}}\right)\right) z_{1}\right)-\prod_{j=\nu+1}^{m}\left(-a_{j} z_{1}\right)\right| \\
& \leq C \max _{\nu+1 \leq k \leq m}\left(|\lambda|+\left|z_{1}\right|\left|a_{k}-\beta_{k}\left(1, \frac{z_{2}}{z_{1}}\right)\right|\right) \cdot\left|\prod_{j \neq k}\left(-a_{j} z_{1}\right)\right| \\
& \leq C\left(\left(M_{0}+\delta\right)\left|z_{2}\right|+\varepsilon\right)\left|z_{1}\right|^{m-\nu-1} \\
& \leq C 2^{m-\nu-1}\left(\left(M_{0}+\delta\right)\left|z_{2}\right|+\varepsilon\right),
\end{aligned}
$$

if $\eta<\sigma(\varepsilon) / 2$. From this and (5.13) we get

$$
\begin{aligned}
\left|\left(F\left(U-Q_{1}\right)\right)\left(z_{1}, z_{2}, \lambda\right)\right| \leq & \left(\delta+M\left|z_{2}\right|\right)\left(\delta+2 M_{0}\right)^{\nu-1} \\
& \times C 2^{m-\nu-1}\left(\left(M_{0}+\delta\right)\left|z_{2}\right|+\varepsilon\right)\left|z_{2}\right|^{\nu} \\
\leq & \frac{1}{16}\left(\frac{\kappa}{2}\right)^{\nu-1} \delta\left|z_{2}\right|^{\nu}
\end{aligned}
$$

if $\varepsilon$ and consequently $\eta$ is small enough. From (5.12) we get

$$
\left|\left(\left(F-Q_{0}\right) Q_{1}\right)\left(z_{1}, z_{2}, \lambda\right)\right| \leq L\left|z_{2}\right|^{\nu+1} \leq \frac{1}{16}\left(\frac{\kappa}{2}\right)^{\nu-1} \delta\left|z_{2}\right|^{\nu},
$$

if $\eta<\frac{1}{16 L}\left(\frac{\kappa}{2}\right)^{\nu-1} \delta$. Combining (5.14) and (5.15) with (5.11) it follows from (5.10) that we can apply Rouché's theorem to conclude that there exist $\rho_{0}>0, R_{1}>0$, and $0<r<\sigma /\left(\delta+M_{0}\right)$ such that the following holds: Whenever $t \geq R_{1}$ and $\left(z_{1}, z_{2}\right) \in \mathbb{C}^{2}$ satisfies $\left|z_{1}\right|<r$ and $t^{-m / \nu} \rho_{0}<$ $\left|z_{2}\right|<r$, to each solution $z=\left(z_{1}, z_{2}, b_{l} z_{2}\right)$ of the equation $Q(\zeta)=0$ there corresponds a unique solution $\left(z_{1}, z_{2}, z_{3}^{l}\left(z_{1}, z\right)\right)$ of the equation $P_{m}(\zeta)=$ $t^{-m}$ satisfying

$$
\left|z_{3}^{l}\left(z_{1}, z_{2}\right)-b_{l} z_{2}\right|<\delta\left|z_{2}\right|
$$


This implies

$$
\left|z_{3}^{l}\left(z_{1}, z_{2}\right)\right|<\delta\left|z_{2}\right|+\left|b_{l} z_{2}\right| \leq\left(\delta+M_{0}\right)\left|z_{2}\right|=D\left|z_{2}\right| \leq D r=s<\sigma
$$

if we let $D:=\delta+M_{0}$ and $s:=D r$. When $z_{1}, z_{2}$ are real, we get for $\zeta:=\left(z_{1}, z_{2}, z_{3}^{l}\left(z_{1}, z_{2}\right)\right)$ :

$$
t^{-m}=\overline{P_{m}(\zeta)}=P_{m}(\bar{\zeta}) \quad \text { and } \quad\left|\bar{\zeta}-b_{l} z_{2}\right|=\left|\bar{\zeta}-\overline{b_{l} z_{2}}\right|<\delta\left|z_{2}\right| \text {, }
$$

since $P_{m}$ has real coefficients and since $b_{l}$ is real. By the uniqueness of the solution, this implies that $z_{3}^{l}\left(z_{1}, z_{2}\right)$ is real for real $z_{1}, z_{2}$. Since the equation $Q\left(z_{1}, z_{2}, \tau\right)=0$ has $\nu$ distinct roots $b_{1} z_{2}, \ldots, b_{\nu} z_{2}$, which are further apart from each other than $\delta\left|z_{2}\right|$, the lemma now follows from an obvious scaling argument.

To apply Lemma 5.6, we need the estimates of the following lemma.

Lemma 5.7. For each $z, w \in \mathbb{C}$ and $\varepsilon>0$ the following inequalities hold:

(i) $\left|\operatorname{Im} \sqrt{z^{2}+w^{2}}\right| \leq|\operatorname{Im} z|+|\operatorname{Im} w|$,

(ii) $|\operatorname{Im} z| \leq\left|\operatorname{Im} \sqrt{z^{2}-\varepsilon^{2}}\right|$.

If, furthermore, $|z| \geq \lambda \varepsilon$ for some $\lambda>1$, then

(iii) $\left|\operatorname{Im} \sqrt{z^{2}-\varepsilon^{2}}\right| \leq \frac{\lambda}{\sqrt{\lambda^{2}-1}}|\operatorname{Im} z|$.

Proof. Let $z=x+i y, w=u+i v$, and $\sqrt{z^{2}+w^{2}}=A+i B$. Then a short calculation shows that

$$
A B=x y+u v \quad \text { and } \quad A^{2}-B^{2}=\left(x^{2}+u^{2}\right)-\left(y^{2}+v^{2}\right) .
$$

Solving for $A$ from the first of these equations and substituting into the second one gives a quadratic equation for $B^{2} \geq 0$ whose solution is

$$
B^{2}=\frac{1}{2}\left\{\left(y^{2}+v^{2}\right)-\left(x^{2}+u^{2}\right)+\left(\left[\left(x^{2}+u^{2}\right)-\left(y^{2}+v^{2}\right)\right]^{2}\right.\right.
$$

16) $\left.\left.\quad+4(x y+u v)^{2}\right)^{\frac{1}{2}}\right\}$.

By the Schwarz inequality, $(x y+u v)^{2} \leq\left(x^{2}+u^{2}\right)\left(y^{2}+v^{2}\right)$, the term under the square-root is not larger than $\left[\left(x^{2}+u^{2}\right)+\left(y^{2}+v^{2}\right)\right]^{2}$, which implies

$$
\left|\operatorname{Im} \sqrt{z^{2}+w^{2}}\right|=|B| \leq \sqrt{y^{2}+v^{2}} \leq|y|+|v|=|\operatorname{Im} z|+|\operatorname{Im} w| .
$$


Hence (i) holds. To prove (ii), set $u=0, v=\varepsilon$ in (5.16) and note that then the term under the square-root is equal to $\left(x^{2}+y^{2}-\varepsilon^{2}\right)^{2}+4 y^{2} \varepsilon^{2}$. If we let $C:=2 y \varepsilon /\left(x^{2}+y^{2}-\varepsilon^{2}\right)$, then we get

$$
B^{2}=\frac{1}{2}\left(y^{2}+\varepsilon^{2}-x^{2}+\left|x^{2}+y^{2}-\varepsilon^{2}\right| \sqrt{1+C^{2}}\right) .
$$

If $x^{2}+y^{2}-\varepsilon^{2} \geq 0$ this implies

$$
\left|\operatorname{Im} \sqrt{z^{2}-\varepsilon^{2}}\right|=|B| \geq|y|=|\operatorname{Im} z|,
$$

since $\sqrt{1+C^{2}} \geq 1$. If $x^{2}+y^{2}-\varepsilon^{2} \leq 0$ then $y^{2}+\varepsilon^{2}-x^{2} \geq 2 y^{2}$ and hence $B^{2} \geq y^{2}$. From this, (ii) follows also in this case.

For the inequality (iii), use the estimate $\sqrt{1+C^{2}} \leq 1+C^{2} / 2$ in (5.17). Since $|z|^{2}=x^{2}+y^{2} \geq \lambda^{2} \varepsilon^{2}$ we obtain

$$
B^{2} \leq y^{2}+y^{2} \frac{\varepsilon^{2}}{x^{2}+y^{2}-\varepsilon^{2}} \leq y^{2}\left(1+\frac{1}{\lambda^{2}-1}\right)=\frac{\lambda^{2} y^{2}}{\lambda^{2}-1} .
$$

Obviously, this implies (iii).

In the following statement, $|\cdot|$ denotes the maximum norm in $\mathbb{C}^{n}$. Everywhere else, the choice of the norm does not matter.

Lemma 5.8. Suppose $0 \leq \varepsilon \leq \frac{1}{2}$ and that $v\left(z^{\prime}, z_{n}\right)$ is plurisubharmonic for $\left|z^{\prime}\right|<1,\left|z_{n}\right|<1$ and satisfies

(i) $v\left(z^{\prime}, z_{n}\right) \leq 1, \quad\left|z^{\prime}\right|<1,\left|z_{n}\right|<1$,

(ii) $v\left(x^{\prime}, x_{n}\right)=0 \quad$ if $x^{\prime}, x_{n}$ are real and $\left|x_{n}\right| \geq \varepsilon$.

Then for each $\lambda<1$ there is a constant $C_{\lambda}$ such that

(iii) $v\left(z^{\prime}, z_{n}\right) \leq C_{\lambda}\left(\left|\operatorname{Im} z^{\prime}\right|+\left|\operatorname{Im} \sqrt{z_{n}^{2}-\varepsilon^{2}}\right|\right), \quad\left|z^{\prime}\right| \leq \lambda,\left|z_{n}\right| \leq \lambda$.

Proof. This follows from standard estimates for harmonic measure, but is perhaps most easily seen using the following explicit formulas. Let $h(\zeta)$ denote the harmonic measure of the real axis in the unit disk. That is, the subharmonic function on $|\zeta|<1$ that is equal to 0 on the real axis, equal to 1 on $|\zeta|=1$, and harmonic for $|\zeta|<1, \operatorname{Im} \zeta \neq 0$. Well-known and easily verified formulas for $h(\zeta), \zeta=x+y$, are $h(\zeta)=\frac{1}{\pi}[\arg (\zeta+1)+\pi-$ $\arg (\zeta-1)]=\frac{2}{\pi} \arctan \frac{|y|}{1-\left(x^{2}+y^{2}\right)}$. Similarly, if $k_{\varepsilon}(\zeta)$ denotes the function harmonic in the unit disk with the real intervals $[-1, \varepsilon],[\varepsilon, 1]$ removed, and with boundary values 1 on $|\zeta|=1$ and 0 on $[-1, \varepsilon] \cup[\varepsilon, 1]$, then

$$
k_{\varepsilon}(\zeta)=h\left(\sqrt{\frac{\zeta^{2}-\varepsilon^{2}}{1-\varepsilon^{2} \zeta^{2}}}\right) .
$$


This is easily seen by considering the sequence of analytic maps $\omega=\zeta^{2}, \tau=$ $\frac{\omega-\varepsilon^{2}}{1-\varepsilon^{2} \omega}, \sigma=\sqrt{\tau}$ which represents $\{|\zeta|<1\} \backslash([-1, \varepsilon] \cup[\varepsilon, 1])$ as a $2-1$ analytic cover of the upper half disk $\{|\sigma|<1, \operatorname{Im} \sigma>0\}$ with $|\zeta|=1$ mapping to $|\sigma|=1$ and the intervals $[-1,-\varepsilon],[\varepsilon, 1]$ to the negative and positive real $\sigma$-axis, respectively.

For $z=\left(z^{\prime}, z_{n}\right) \in \mathbb{C}^{n}$ with $\left|z^{\prime}\right|<1$ and $\left|z_{n}\right|<1$, the maximum principle easily shows $v(z) \leq h\left(z_{1}\right)+\cdots+h\left(z_{n-1}\right)+k_{\epsilon}\left(z_{n}\right)$ for every plurisubharmonic function $v$ satisfying (i) and (ii). Since $h(\zeta) \leq C_{\lambda}|\operatorname{Im} \zeta|$ and $k_{\varepsilon}(\zeta) \leq C_{\lambda} \mid \operatorname{Im} \sqrt{\zeta^{2}-\varepsilon^{2}}$ for $|\zeta| \leq \lambda<1$, it follows that (iii) also holds.

Lemma 5.9. Assume that $P_{m}$ and $\xi=(1,0,0)$ satisfy the hypotheses of Lemma 5.6 and let $\rho_{0} \geq 1, r, s>0$, and $R_{1} \geq 1$ be the constants which exist by that lemma. Then there exist constants $A_{1}, A_{2}, A_{3} \geq 1$ such that for each $t \geq R_{1}$ the following holds: Whenever $u \in \operatorname{PSH}\left(V_{ \pm}\left(P_{m}\right)\right)$ satisfies the conditions $(\alpha)$ and $(\beta)$ of $2.2(\mathrm{a})$, then for $z \in V_{ \pm}\left(P_{m}\right) \cap B(t \xi ; r t / 2, r t / 2$, st $/ 2)$ we have the estimates

(a) $u\left(z_{1}, z_{2}, z_{3}\right) \leq A_{1}\left|\operatorname{Im} z_{1}\right|+A_{2} t^{1-m / \nu}$ if $\left|z_{2}\right| \leq 4 \rho_{0} t^{1-m / \nu}$,

(b) $u\left(z_{1}, z_{2}, z_{3}\right) \leq A_{1}\left|\operatorname{Im} z_{1}\right|+A_{3}\left|\operatorname{Im} z_{2}\right|$ if $\left|z_{2}\right|>2 \rho_{0} t^{1-m / \nu}$.

Proof. Since the arguments for $V_{+}\left(P_{m}\right)$ and $V_{-}\left(P_{m}\right)$ are the same, we only treat $V_{+}\left(P_{m}\right)$. Fix $u \in \operatorname{PSH}\left(V_{+}\left(P_{m}\right)\right)$ satisfying $(\alpha)$ and $(\beta)$ of $2.2($ a). Since $0 \notin V_{+}\left(P_{m}\right)$ it follows from Theorem 5.2 that there exists $A_{0}^{\prime} \geq 1$, not depending on $u$, such that

$$
u(z) \leq A_{0}^{\prime}|z|, \quad z \in V_{+}\left(P_{m}\right) .
$$

It is no restriction to assume in the sequel $r \leq \frac{1}{2}$ and $R_{1} \geq 16 \rho_{0} / r$. Next fix $t \geq R_{1}$ and define $v: B((t, 0) ; r t) \rightarrow[-\infty, \infty[$ by

$$
v\left(z_{1}, z_{2}\right):=\max \left\{u\left(z_{1}, z_{2}, z_{3}\right): P_{m}\left(z_{1}, z_{2}, z_{3}\right)=1,\left|z_{3}\right|<s t\right\} .
$$

Then $v$ is plurisubharmonic and by Lemma 5.6 it satisfies

$$
v\left(z_{1}, z_{2}\right) \leq 0 \quad \text { if } \quad z_{1}, z_{2} \in \mathbb{R} \quad \text { and } \quad \rho_{0} t^{1-m / \nu} \leq\left|z_{2}\right|<r t .
$$

Further, we get from Lemma 5.6 and (5.18)

$$
\begin{aligned}
v\left(z_{1}, z_{2}\right) & \leq A_{0}^{\prime}\left(\left|\left(z_{1}, z_{2}\right)\right|+\max _{1 \leq j \leq \nu}\left|z_{3}^{j}\left(z_{1}, z_{2}\right)\right|\right) \\
& \leq A_{0}^{\prime}(1+D)\left|\left(z_{1}, z_{2}\right)\right| \leq A_{0}\left|\left(z_{1}, z_{2}\right)\right| \\
& \leq A_{0}(1+2 r) t \leq 3 A_{0} t \text { for }\left(z_{1}, z_{2}\right) \in B((t, 0) ; r t),
\end{aligned}
$$

where $A_{0}:=A_{0}^{\prime}(1+D)$. Now let

$$
\varphi\left(\zeta^{\prime}, \zeta_{2}\right):=\frac{1}{3 A_{0} t} v\left(r t\left(\zeta^{\prime}+1\right), r, t \zeta_{2}\right),\left(\zeta^{\prime}, \zeta\right) \in B(0 ; 1) .
$$


The properties of $v$ imply that $\varphi$ is plurisubharmonic on $B(0 ; 1)$, bounded by 1 , and equal to 0 if $\zeta^{\prime}, \zeta_{2}$ are real and $\left|\zeta_{2}\right| \geq \varepsilon:=\rho_{0} /\left(r t^{m / \nu}\right)$. Hence we can apply Lemma 5.8 to $\varphi$ to conclude that there is a constant $A_{1}$, independent of $v$, such that

$$
\begin{aligned}
& v\left(z_{1}, z_{2}\right) \leq A_{1}\left(\left|\operatorname{Im} z_{1}\right|+\left|\operatorname{Im} \sqrt{z_{2}^{2}-\left(\rho_{0} t^{1-m / \nu}\right)^{2}}\right|\right), \\
& \left(z_{1}, z_{2}\right) \in B((t, 0) ; r t / 2) .
\end{aligned}
$$

The estimates (a) and (b) are now easy consequences of (5.21), part (a) by applying Lemma 5.7(i) and part (b) from Lemma 5.7(iii).

To formulate our next lemma, we introduce the following notation for cones with truncated tip. If $\xi \in \mathbb{C}^{n},|\xi|=1, \delta>0$, and $R \geq 0$ are given we let

$$
\Gamma(\xi, \delta, R):=\left\{z \in \mathbb{C}^{n}:\left|\frac{z}{|z|}-\xi\right|<\delta, \quad|z|>R\right\} .
$$

Lemma 5.10. Let $P_{m} \in \mathbb{R}\left[z_{1}, z_{2}, z_{3}\right]$ be homogeneous of degree $m \geq 2$ and assume that $P_{m}$ satisfies condition (4) of Theorem 5.1. Then for each $\xi \in V\left(P_{m}\right) \cap \mathbb{R}^{3},|\xi|=1$, there exist $\delta_{\xi}>0, R_{\xi} \geq 1$, and $A_{\xi} \geq 1$ such that each $u \in \operatorname{PSH}\left(V_{ \pm}\left(P_{m}\right)\right)$ which satisfies the conditions $(\alpha)$ and $(\beta)$ of 2.2(a), also satisfies

$$
u(z) \leq A_{\xi}|\operatorname{Im} z| \quad \text { for } z \in V_{ \pm}\left(P_{m}\right) \cap \Gamma\left(\xi, \delta_{\xi}, R_{\xi}\right) .
$$

Proof. By hypothesis, we can apply Lemma 5.3. In particular, we can assume $\xi=(1,0,0)$. Then we apply Lemma 5.6 and Lemma 5.9 to get $\rho_{0} \geq 1$, $\frac{1}{2} \geq r>0, s>0, R_{1} \geq 1$, and constants $A_{1}, A_{2}, A_{3}$ such that the conclusion of Lemma 5.9 holds. To prove the present lemma it suffices to show that there exist $\delta>0, R_{2} \geq R_{1}$, and $A \geq 1$ such that each $u \in \operatorname{PSH}\left(V_{ \pm}\left(P_{m}\right)\right)$ which satisfies the conditions $(\alpha)$ and $(\beta)$ of 2.2(a) also satisfies

$$
u(z) \leq A|\operatorname{Im} z| \quad \text { for } \quad z \in \bigcup_{t>R_{2}} t B(\xi ; \delta) .
$$

Note that by Lemma 5.9(b), the estimate (5.22) holds with $A=\max \left(A_{1}, A_{3}\right)$ on the set $\bigcup_{t>R_{1}}\left\{z \in t B(\xi ; r / 2, r / 2, \sigma / 2):\left|z_{2}\right|>2 \rho_{0} t^{-m / \nu}\right\}$. To prove (5.22) on $\bigcup_{t>R_{2}}\left\{z \in t B(\xi ; \delta):\left|z_{2}\right|<4 \rho_{0} t^{-m / \nu}\right\}$, for suitable $\delta>0$ and $R_{2} \geq R_{1}$, we will use the notation introduced in the proof of Lemma 5.6. Then we have for $z \in B(\xi ; \eta, \eta, \sigma)$

$$
\begin{aligned}
& P_{m}(z)=F(z) U(z), \quad F(z)=\prod_{j=1}^{\nu}\left(z_{3}-b_{j} z_{2}-g_{j}\left(z_{1}, z_{2}\right)\right), \\
& U(z)=\frac{P_{m}(z)}{F(z)}
\end{aligned}
$$


As in the proof of Lemma 5.6 we assume without restriction that $U(1,0,0)=$ $\prod_{j=\nu+1}^{m}\left(-a_{j}\right)=1$. Then it is easy to check that for the localization at $\xi$ we have

$$
\begin{aligned}
& \left(P_{m}\right)_{\xi}=(F)_{\xi}(U)_{\xi}=(F)_{\xi}=Q_{0}, \quad \text { where } \\
& Q_{0}\left(z_{2}, z_{3}\right)=\prod_{j=1}^{\nu}\left(z_{3}-b_{j} z_{2}\right) .
\end{aligned}
$$

Subsequently we will only treat the case $V_{+}\left(P_{m}\right)$, since the same arguments apply to $V_{-}\left(P_{m}\right)$. To do so note that by Lemma 3.10 ,

$$
S(\tau, z):=P_{m}\left(\frac{z_{1}}{\tau^{\nu}}, z_{2} \tau^{m-\nu}, z_{3} \tau^{m-\nu}\right)-1
$$

is a polynomial in $\left(\tau, z_{1}, z_{2}, z_{3}\right)$ which satisfies

$$
S(0, z)=z_{1}^{m-\nu}\left(P_{m}\right)_{\xi}\left(z_{2}, z_{3}\right)=z_{1}^{m-\nu} Q_{0}\left(z_{2}, z_{3}\right) .
$$

Since the real numbers $b_{1}, \ldots, b_{\nu}$ are distinct, $\operatorname{grad} Q_{0}\left(z_{2}, z_{3}\right) \neq 0$ for $\left(z_{2}, z_{3}\right) \neq 0$. Hence

$$
\begin{aligned}
& \frac{\partial S}{\partial z_{2}}(0, z) \neq 0 \quad \text { or } \quad \frac{\partial S}{\partial z_{3}}(0, z) \neq 0 \\
& \text { if } \quad z_{1} \neq 0 \quad \text { and } \quad\left(z_{2}, z_{3}\right) \neq 0 .
\end{aligned}
$$

Next let $0<r \leq \eta, 0<s \leq \sigma$ be the numbers which exist by Lemma 5.6 and note that

$$
K:=\left\{(0, z) \in \mathbb{R}^{4}: z \in \overline{B(\xi ; r / 2, r / 2, s / 2)}, S(0, z)=0\right\}
$$

is compact. Since $(0,0) \notin K$, for each $(0, z) \in K$ we have $\frac{\partial S}{\partial z_{2}}(0, z) \neq 0$ or $\frac{\partial S}{\partial z_{3}}(0, z) \neq 0$. We let

$$
M_{j}:=\left\{z:(0, z) \in K, \frac{\partial S}{\partial z_{j}}(0, z) \neq 0\right\}, \quad j=2,3 .
$$

Then for each $z \in M_{3}$ the implicit function theorem implies the existence of $\delta(z)=\left(\delta_{0}(z), \ldots, \delta_{3}(z)\right)>0$ and of a holomorphic function $h_{3}^{z}$ : $B\left(\left(0, z_{1}, z_{2}\right) ; \delta^{\prime}(z)\right) \rightarrow B\left(z_{3} ; \delta_{3}(z)\right)$ such that

$$
\begin{aligned}
V(S) \cap B((0, z) ; \delta(z))= & \left\{\left(\tau, \zeta_{1}, \zeta_{2}, h_{3}^{z}\left(\tau, \zeta_{1}, \zeta_{2}\right)\right):\left(\tau, \zeta_{1}, \zeta_{2}\right)\right. \\
& \left.\in B\left(\left(0, z_{1}, z_{2}\right) ; \delta^{\prime}(z)\right)\right\}
\end{aligned}
$$


Since $(0, z) \in \mathbb{R}^{4} \cap V(S)$, the real implicit function theorem implies that $h_{3}^{z}\left(\tau, \zeta_{1}, \zeta_{2}\right)$ is real when $\left(\tau, \zeta_{1}, \zeta_{2}\right)$ is real. The same arguments apply to the points $z \in M_{2}$. Now note that

$$
\begin{aligned}
& \left\{B\left((0, z) ; \frac{\delta_{0}(z)}{2}, \frac{\delta_{1}(z)}{2}, \delta_{2}(z), \frac{\delta_{3}(z)}{2}\right): z \in M_{2}\right\} \cup \\
& \quad\left\{B\left((0, z) ; \frac{\delta_{0}(z)}{2}, \frac{\delta_{1}(z)}{2}, \frac{\delta_{2}(z)}{2}, \delta_{3}(z)\right): z \in M_{3}\right\}
\end{aligned}
$$

is an open cover of $K$. Hence there exist $z_{1}, \ldots, z_{k} \in M_{2}$ and $z_{k+1}, \ldots, z_{l} \in$ $M_{3}$ such that the elements of the cover with centers $\left(0, z_{1}\right), \ldots,\left(0, z_{l}\right)$ cover $K$. Let $\delta_{0}:=\min \left\{\delta_{0}\left(z_{j}\right): 1 \leq j \leq l\right\}$. Then

$$
\begin{aligned}
U:= & \bigcup_{j=1}^{k} B\left(\left(0, z_{j}\right) ; \frac{\delta_{0}}{2}, \frac{\delta_{1}\left(z_{j}\right)}{2}, \delta_{2}\left(z_{j}\right), \frac{\delta_{3}\left(z_{j}\right)}{2}\right) \\
& \cup \bigcup_{j=k+1}^{l} B\left(\left(0, z_{j}\right) ; \frac{\delta_{0}}{2}, \frac{\delta_{1}\left(z_{j}\right)}{2}, \frac{\delta_{2}\left(z_{j}\right)}{2}, \delta_{3}\left(z_{j}\right)\right)
\end{aligned}
$$

is a neighborhood of $K$. Hence

$$
L_{0}:=(\overline{B((0, \xi) ; 1, r / 2, r / 2, s / 2)} \backslash U) \cap V(S) \cap \mathbb{R}^{4}
$$

is compact and so is its projection in the $\tau$-space. Therefore

$$
\varepsilon_{1}:=\inf \left\{\tau:\left(\tau, z_{1}, z_{2}, z_{3}\right) \in L_{0}\right\}
$$

is obtained at some point $\left(\tau_{0}, w_{1}, w_{2}, w_{3}\right) \in L_{0}$. If we assume $\tau_{0}=0$, then $\left(0, w_{1}, w_{2}, w_{3}\right)$ is in $K$, in contradiction to the definition of $L_{0}$. This proves $\varepsilon_{1}>0$.

Next note that also

$$
L_{1}:=(\overline{B((0, \xi) ; 1, r / 2, r / 2, s / 2)} \backslash U) \cap V(S) \cap\left(\left[0, \varepsilon_{1} / 2\right] \times \mathbb{C}^{3}\right)
$$

is compact. Hence

$$
\varepsilon_{2}:=\inf \left\{|\operatorname{Im} z|:(\tau, z) \in L_{1}\right\}
$$

is obtained at some point $\left(\tau_{1}, \zeta\right) \in L_{1}$. If we assume $\varepsilon_{2}=0$, then $\left(\tau_{1}, \zeta\right)$ is real and hence in $L_{0}$. By the definition of $\varepsilon_{1}$, this implies $\tau_{1} \geq \varepsilon_{1}$, in contradiction to $\tau_{1} \in\left[0, \varepsilon_{1} / 2\right]$. This proves $\varepsilon_{2}>0$.

Now choose $R_{2} \geq \max \left(\left(2 / \varepsilon_{1}\right)^{\nu}, R_{1}\right)$ so large that $\max _{1 \leq j \leq l} 4 /\left(\pi \delta_{1}\left(z_{j}\right)\right.$ $\left.\left(A_{1}+A_{2}\right)\right) \leq R_{2}^{m / \nu}$, and fix $\tau \geq R_{2}$ and $\zeta \in V_{+}\left(P_{m}\right) \cap t B(\xi, r / 4, r / 4, s / 4)$ satisfying $\left|\zeta_{2}\right| \leq 4 \rho_{0} t^{1-m / \nu}$. Then let

$$
\tau:=\frac{1}{t^{1 / \nu}}, z_{1}:=\zeta_{1} \tau^{\nu}, z_{2}:=\zeta_{2} \tau^{\nu-m}, z_{3}:=\zeta_{3} \tau^{\nu-m} .
$$


It is easy to check that $(\tau, z) \in V(S) \cap B\left((0, \xi) ; \varepsilon_{1} / 2, r / 4, r / 4, s / 4\right)$. Now we fix $u \in \operatorname{PSH}\left(V_{+}\left(P_{m}\right)\right)$ satisfying the conditions $(\alpha)$ and $(\beta)$ of 2.2(a). As we have seen in the proof of Lemma 5.9, there exists $A_{0}^{\prime} \geq 1$ such that (5.18) holds. Now we distinguish the following cases:

Case $(\tau, z) \notin U$ Since $\tau \in\left[0, \varepsilon_{1} / 2\right]$, the point $(\tau, z)$ is in $L_{1}$. By the definition of $\varepsilon_{2}$, this implies $|\operatorname{Im} z| \geq \varepsilon_{2}$.

Case $(\tau, z) \notin U,\left|\operatorname{Im} z_{1}\right| \geq \varepsilon_{2} / 3$ Note first that $\frac{1}{2} t \leq\left|\zeta_{1}\right| \leq 2 t$, since $0<r \leq \frac{1}{2}$. Hence the present hypothesis implies

$$
\left|\operatorname{Im} \zeta_{1}\right|=\tau^{-\nu}\left|\operatorname{Im} z_{1}\right| \geq \frac{\varepsilon_{2}}{3} t \geq \frac{\varepsilon_{2}}{6}\left|\zeta_{1}\right| .
$$

From this and (5.18) we get

$$
\begin{aligned}
u(\zeta) \leq A_{0}^{\prime}|\zeta| & \leq A_{0}^{\prime}\left(1+\frac{r}{2}+\frac{s}{4}\right) t \\
& \leq 12 A_{0}^{\prime}\left(1+\frac{r}{2}+\frac{s}{4}\right) \frac{1}{\varepsilon_{2}}|\operatorname{Im} \zeta| .
\end{aligned}
$$

Case $(\tau, z) \notin U,\left|\operatorname{Im}\left(z_{2}, z_{3}\right)\right| \geq \varepsilon_{2} / 3$ Then we have

$$
\left|\operatorname{Im}\left(\zeta_{2}, \zeta_{3}\right)\right|=\tau^{m-\nu}\left|\operatorname{Im}\left(z_{2}, z_{3}\right)\right| \geq \frac{\varepsilon_{2}}{3} t^{1-m / \nu} .
$$

From this and Lemma 5.9(a) we get

$$
\begin{aligned}
u(\zeta) & \leq A_{1}\left|\operatorname{Im} \zeta_{1}\right|+A_{2} \frac{3}{\varepsilon_{2}}\left|\operatorname{Im}\left(\zeta_{2}, \zeta_{3}\right)\right| \\
& \leq\left(A_{1}+\frac{3 A_{2}}{\varepsilon_{2}}\right)|\operatorname{Im} \zeta| .
\end{aligned}
$$

Case $(\tau, z) \in U$ Without restriction we assume that there exists $w \in\left\{z_{k+1}\right.$, $\left.\ldots, z_{l}\right\}$ such that $(\tau, z) \in V(S) \cap B\left((0, w) ; \frac{\delta_{0}}{2}, \frac{\delta_{1}(w)}{2}, \frac{\delta_{2}(w)}{2}, \delta_{3}(w)\right)$. By the choice of $\delta(w)$, we know that $V(S) \cap B((0, w) ; \delta(w))$ is the graph of a holomorphic function $h: B\left(\left(0, w_{1}, w_{2}\right) ; \delta_{0}(w), \delta_{1}(w), \delta_{2}(w)\right) \rightarrow \mathbb{C}$ which is real when $\left(v, y_{1}, y_{2}\right) \in B\left(\left(0, w_{1}, w_{2}\right) ; \delta_{0}(w), \delta_{1}(w), \delta_{2}(w)\right) \cap$ $\mathbb{R}^{3}$. Now note that the definition of $S$ implies that for $\left(y_{1}, y_{2}\right) \in B\left(\left(w_{1}\right.\right.$, $\left.\left.w_{2}\right) ; \delta_{1}(w), \delta_{2}(w)\right)$ the point $\left(\tau^{-\nu} y_{1}, \tau^{m-\nu} y_{2}, \tau^{m-\nu} h\left(\tau, y_{1}, y_{2}\right)\right)$ is in $V_{+}\left(P_{m}\right)$. Therefore, we can define a plurisubharmonic function $\varphi$ on $B\left(\left(w_{1}, w_{2}\right) ; \delta_{1}(w), \delta_{2}(w)\right)$ by

$$
\varphi\left(y_{1}, y_{2}\right)=u\left(\tau^{-\nu} y_{1}, \tau^{m-\nu} y_{2}, t^{m-\nu} h\left(\tau, y_{1}, y_{2}\right)\right) .
$$

By Lemma 5.9(a) and the properties of $h$ we get

$$
\begin{aligned}
& \varphi\left(y_{1}, y_{2}\right) \leq\left(A_{1}+A_{2}\right)\left(\tau^{-\nu}\left|\operatorname{Im} y_{1}\right|+\tau^{m-\nu}\right) \quad \text { and } \\
& \varphi\left(y_{1}, y_{2}\right) \leq 0 \text { if }\left(y_{1}, y_{2}\right) \in \mathbb{R}^{2} .
\end{aligned}
$$


Now fix $y_{2}$ real and consider for $\operatorname{Im} y_{1} \geq 0$ the function

$$
\psi: y_{1} \mapsto \varphi\left(y_{1}, y_{2}\right)-\left(A_{1}+A_{2}\right) \tau^{-\nu} \operatorname{Im} y_{1} .
$$

Then we have

$$
\psi\left(y_{1}\right) \leq\left(A_{1}+A_{2}\right) \tau^{m-\nu} \quad \text { and } \quad \varphi\left(y_{1}\right) \leq 0 \text { if } y_{1} \in \mathbb{R} .
$$

From this and an application of the standard estimate for the harmonic measure of the real line in the unit disk it follows as in the proof of Lemma 5.8 that

$$
\psi\left(y_{1}\right) \leq \frac{4}{\pi}\left(A_{1}+A_{2}\right) \frac{2}{\delta_{1}(w)} \tau^{m-\nu}\left|\operatorname{Im} y_{1}\right|, \quad y_{1} \in B\left(w_{1} ; \delta_{1}(w) / 2\right) .
$$

Arguing similarly for $\operatorname{Im} y_{1} \leq 0$, we conclude for $y_{2}$ real:

$$
\begin{aligned}
\varphi\left(y_{1}, y_{2}\right) & \leq\left(A_{1}+A_{2}\right) \tau^{-\nu}\left|\operatorname{Im} y_{1}\right|\left(1+\frac{8 \tau^{m}}{\pi \delta_{1}(w)}\right) \\
& \leq 2\left(A_{1}+A_{2}\right) \tau^{-\nu}\left|\operatorname{Im} y_{1}\right|,
\end{aligned}
$$

by our choice of $R_{2}$. Next we fix $y_{1}$ and argue in the same way to get

$$
\begin{aligned}
\varphi\left(y_{1}, y_{2}\right) \leq & 2\left(A_{1}+A_{2}\right) \tau^{-\nu}\left|\operatorname{Im} y_{1}\right| \\
& +\left(A_{1}+A_{2}\right) \frac{8}{\pi \delta_{2}(w)} \tau^{m-\nu}\left|\operatorname{Im} y_{2}\right|
\end{aligned}
$$

for $\left(y_{1}, y_{2}\right) \in B\left(\left(w_{1}, w_{2}\right) ; \delta_{1}(w) / 2, \delta_{2}(w) / 2\right)$. In particular, we get

(5.25) $u\left(\zeta_{1}, \zeta_{2}, \zeta_{3}\right)=\varphi\left(z_{1}, z_{2}\right) \leq 2\left(A_{1}+A_{2}\right)\left(\left|\operatorname{Im} \zeta_{1}\right|+\left|\operatorname{Im} \zeta_{2}\right|\right)$,

provided that $R_{2}$ is large enough.

Now it follows from (5.25), (5.26), and (5.27) that (5.24) holds provided that we choose $\delta>0$ small and $R_{2}$ and $A \geq 1$ large enough.

As we have noted at the beginning, this completes the proof.

After all these preparations we can finally prove Theorem 5.1.

Proof of Theorem 5.1. (1) $\Rightarrow$ (2): By Lemma 2.5, the hypothesis implies that $V\left(P_{m}\right)$ has $\mathrm{PL}\left(\mathbb{R}^{3}, \log \right)$. Hence $P_{m}$ is real up to a complex constant by Meise, Taylor, and Vogt [13], Lemma 2. If we assume $P_{m}=\lambda Q_{m}$ for some $\lambda \in \mathbb{C} \backslash \mathbb{R}$ and $Q_{m} \in \mathbb{R}\left[z_{1}, z_{2}, z_{3}\right]$, then $V_{+}\left(P_{m}\right)=\left\{z \in \mathbb{C}^{3}: Q_{m}(z)=\right.$ $1 / \lambda\}$. Since $Q_{m}$ has real coefficients and $1 / \lambda \notin \mathbb{R}, V_{+}\left(P_{m}\right) \cap \mathbb{R}^{3}=\emptyset$. Obviously, this implies that $V_{+}\left(P_{m}\right)$ fails (SPL) in contradiction to the present hypothesis. Hence $P_{m}$ has real coefficients. Therefore (2) follows from (1) by the result of Meise and Taylor [9], Theorem 3.4, which we recalled in Theorem 2.7. 
(2) $\Rightarrow$ (3): This holds by Lemma 2.5(a).

(3) $\Rightarrow(4)$ : Let $\widetilde{P}_{m}$ denote the polynomial $P_{m}$, regarded as an element of $\mathbb{C}\left[z_{1}, \ldots, z_{4}\right]$, and note that $\widetilde{P}_{m}$ is the principal part of $P$. By Meise, Taylor, and Vogt [15], Theorem 4.1, $V\left(\widetilde{P}_{m}\right)$ has $\operatorname{PL}\left(\mathbb{R}^{4}, \log \right)$. Since $V\left(\widetilde{P}_{m}\right)=$ $V\left(P_{m}\right) \times \mathbb{C}$, this implies that $V\left(P_{m}\right)$ satisfies $\mathrm{PL}\left(\mathbb{R}^{3}, \log \right)$ and hence (SPL) by Lemma 2.5. As in the proof of $(1) \Rightarrow(2), P_{m}$ must be real up to a complex constant. However, by Meise and Taylor [9], Lemma 2.1, a polynomial of the form $P_{m}\left(z_{1}, \ldots, z_{n}\right)+\lambda z_{n+1}$ where $P_{m}$ has real coefficients cannot satisfy $\operatorname{PL}\left(\mathbb{R}^{4}, \log \right)$ unless $\lambda$ is also real. Therefore $P_{m}$ must have real coefficients. Then the last condition in (4) follows from (3) by Theorem 4.1.

$(4) \Rightarrow(1)$ : We will only prove that $V_{+}\left(P_{m}\right)$ satisfies (SPL), since one can argue in the same way for $V_{-}\left(P_{m}\right)$. To do so, Proposition 4.5 of Meise and Taylor [9] will be applied. This result shows that $V_{+}\left(P_{m}\right)$ satisfies (SPL) if and only if the following three conditions hold:

(i) $V_{+}\left(P_{m}\right)$ satisfies $\mathrm{PL}_{\text {loc }}(\xi)$ at each $\xi \in V_{+}\left(P_{m}\right) \cap \mathbb{R}^{3}$,

(ii) there are constants $A, B>0$ such that $u(z) \leq A|z|+B$ for all plurisubharmonic functions $u$ on $V_{+}\left(P_{m}\right)$ satisfying $u(z) \leq|z|+o(|z|)$ on $V$ and $u(z) \leq 0$ on $V_{+}\left(P_{m}\right) \cap \mathbb{R}^{3}$,

(iii) for each $\xi \in V\left(P_{m}\right) \cap \mathbb{R}^{n},|\xi|=1$, there exist $A_{\xi}, \delta_{\xi}$, and $R_{\xi}>0$ such that each $u \in \operatorname{PSH}\left(V_{+}\left(P_{m}\right)\right)$ which satisfies the conditions $(\alpha)$ and $(\beta)$ of 2.2(a), also satisfies $u(z) \leq A_{\xi}|\operatorname{Im} z|$ for $z \in V_{+}\left(P_{m}\right) \cap \Gamma\left(\xi, \delta_{\xi}, R_{\xi}\right)$.

To check these conditions, note first that $V_{+}\left(P_{m}\right)$ is a manifold at each point by Euler's rule. That is,

$$
\sum_{j=1}^{3} z_{j} \frac{\partial P_{m}}{\partial z_{j}}(z)=m P_{m}(z)=m, \quad z \in V_{+}\left(P_{m}\right),
$$

so $\operatorname{grad} P_{m}(z) \neq 0$ for each $z \in V_{+}\left(P_{m}\right)$. Hence $\mathrm{PL}_{\text {loc }}(\xi)$ holds at each point of $V_{+}\left(P_{m}\right)$ as we have noted in Remark 3.2. Thus condition (i) holds. Next note that the hypotheses in (4) obviously imply that $P_{m}$ is square-free. Hence condition (ii) follows from Theorem 5.2 applied to $Q(z)=-1$. Since condition (iii) holds by Lemma 5.10 , we conclude that $V_{+}\left(P_{m}\right)$ satisfies (SPL).

Remark. Let $P_{m} \in \mathbb{R}\left[z_{1}, \ldots, z_{n}\right]$ be homogeneous of degree $m$. By Meise and Taylor [9], Theorem 4.8, $V_{ \pm}\left(P_{m}\right)$ satisfies (SPL) whenever $P_{m}$ is of principal type (i.e., $\sum_{j=1}^{n}\left|\frac{\partial P_{m}}{\partial z_{j}}(\xi)\right|^{2} \neq 0$ for $\xi \in \mathbb{R}^{n} \backslash\{0\}$ ) and each irreducible factor of $P_{m}$ has at least one nontrivial real zero. For $n=2$ this sufficient condition is also necessary and equivalent to $P_{m}$ being strictly hyperbolic. Note that in several steps of the proof of Theorem 5.1 we have used that $\left(P_{m}\right)_{\theta}$ is hyperbolic at each $\theta \in V\left(P_{m}\right) \cap \mathbb{R}^{3},|\theta|=1$. For $n=3$, Theorem 5.1 shows that the condition that $P_{m}$ is of principal type is not 
necessary, since $V\left(P_{m}\right) \cap \mathbb{R}^{3}$ may contain non-zero singular points, as the following examples show.

Example 5.11. (a) For $P_{3}(x, y, z):=y^{2} z-x(x-z)^{2}$ the variety $V\left(P_{3}\right)$ is singular exactly at the points $\{(\lambda, 0, \lambda): \lambda \in \mathbb{C}\}$ and the varieties $V_{ \pm}\left(P_{3}\right)$ satisfy (SPL).

To prove this, note first that

$$
\operatorname{grad} P_{3}(x, y, z)=\left(-3 x^{2}+4 x z-z^{2}, 2 y z, y^{2}+2 x^{2}-2 x z\right) \text {. }
$$

From this it follows easily that

$$
\left\{\xi \in \mathbb{C}^{3}: P_{3}(\xi)=0 \text { and } \operatorname{grad} P_{3}(\xi)=0\right\}=\{(\lambda, 0, \lambda): \lambda \in \mathbb{C}\} .
$$

This proves the first assertion. To prove the second one we show that the conditions in 5.1(4) are satisfied. Obviously, $P_{3}$ has real coefficients. To show that $V\left(P_{3}\right)$ satisfies $\mathrm{PL}\left(\mathbb{R}^{3}, \log \right)$ and hence $(\mathrm{SPL})$, note first that a simple computation shows for $\theta=(1,0,1)$

$$
\left(P_{3}\right)_{\theta}(x, y, z)=y^{2}-(x-z)^{2} .
$$

Hence $\left(P_{3}\right)_{\theta}$ is the product of two distinct real linear forms and therefore square-free. Moreover, $P_{3}$ is locally hyperbolic in the sense of Andersson (see Hörmander [8], 6.4). Thus it follows from Hörmander [8], Theorem 6.5, that $P_{3}(D)$ is a surjective differential operator from the space of all analytic functions on $\mathbb{R}^{3}$ into itself. Since $P_{3}$ is irreducible and not elliptic, by Meise, Taylor, and Vogt [15], Corollary 3.14, and Lemma 2.5 it follows that $V\left(P_{3}\right)$ has (SPL). Therefore, Theorem 5.1 implies that $V_{+}\left(P_{3}\right)$ and $V_{-}\left(P_{3}\right)$ satisfy (SPL).

Note that $\left\{(x, y) \in \mathbb{R}^{2}: P_{3}(x, y, 1)=0\right\}$ is a well-known algebraic curve, namely Newton's knot.

(b) For $P_{3}(x, y, z)=x^{3}+y^{3}-3 x y z$ the variety $V\left(P_{3}\right)$ is singular exactly at the points $\{(0,0, \lambda): \lambda \in \mathbb{C}\}$ and the varieties $V_{ \pm}\left(P_{m}\right)$ both satisfy (SPL).

This can be shown similarly as in part (a). Note that $\left\{(x, y) \in \mathbb{R}^{2}\right.$ : $\left.P_{3}(x, y, 1)=0\right\}$ is the Cartesian leaf.

The following example shows that the Phragmén-Lindelöf property is not inherited by quasihomogeneous principal parts.

Example 5.12. Set $P\left(z_{1}, \ldots, z_{4}\right)=z_{1} z_{2}\left(z_{3}^{2}-z_{1}^{2}\right)-z_{4}$. By Theorem 5.1, its variety $V(P)$ satisfies $\operatorname{PL}\left(\mathbb{R}^{4}, \log \right)$. Define the weight $d=(1,1,2,6)$, and denote by $Q(z)=z_{1} z_{2} z_{3}^{2}-z_{4}$ the $d$-quasihomogeneous principal part of $P$. The principal part $Q_{4}$ of $Q$ is not square-free, hence by Corollary 4.6, the variety $V(Q)$ does not satisfy $\operatorname{PL}\left(\mathbb{R}^{4}, \log \right)$. This shows that $\operatorname{PL}\left(\mathbb{R}^{n}, \omega\right)$ is not inherited by quasihomogeneous principal parts. Note that the classical principal part $P_{m}$ of $P$ satisfies $\operatorname{PL}\left(\mathbb{R}^{n}, \omega\right)$ whenever $P$ does, as Meise, Taylor, and Vogt have shown in [15], 4.1. 


\section{References}

1. Braun, R.W.: Hörmander's Phragmén-Lindelöf principle and irreducible singularities of codimension 1, Boll. Un. Mat. Ital. (7) 6-A(1992), 339-348.

2. Braun, R.W.: The surjectivity of a constant coefficient homogeneous differential operator on the real analytic functions and the geometry of its symbol, Ann. Inst. Fourier (Grenoble), 45 (1995), 223-249.

3. Braun, R.W., Meise, R., Taylor B.A.: Ultradifferentiable functions and Fourier analysis, Res. Math. 17 (1990), 207-237.

4. Braun, R.W., Meise, R., Taylor B.A.: Characterization of the homogeneous polynomials $P$ for which $(P+Q)(D)$ admits a continuous linear right inverse for all lower order perturbations $Q$, Pacific J. Math., to appear.

5. Braun, R.W., Meise, R., Taylor B.A.: A radial Phragmén-Lindelöf estimate for plurisubharmonic functions on algebraic varieties, preprint.

6. Braun, R.W., Meise, R., Vogt, D.: Characterization of the linear partial differential operators with constant coefficients which are surjective on non-quasianalytic classes of Roumieu type on $\mathbb{R}^{n}$, Math. Nachr. 168 (1994), 19-54.

7. Franken, U., Meise, R.: Extension and lacunas of solutions of linear partial differential equations, Ann. Inst. Fourier (Grenoble) 46 (1996), 429-464.

8. Hörmander, L.: On the existence of real analytic solutions of partial differential equations with constant coefficients, Invent. Math. 21 (1973), 151-183.

9. Meise, R., Taylor, B.A.: Phragmén-Lindelöf conditions for graph varieties, to appear in Res. Math.

10. Meise, R., Taylor, B.A., Vogt, D.: Charactérisation des opérateurs linéaires aux dérivées partielles avec coefficients constants sur $\mathcal{E}\left(\mathbb{R}^{n}\right)$ admettant un inverse à droite qui est linéaire et continu, C. R. Acad. Paris 307 (1988), 239-242.

11. Meise, R., Taylor, B.A., Vogt, D.: Characterization of the linear partial operators with constant coefficients that admit a continuous linear right inverse, Ann. Inst. Fourier (Grenoble), 40 (1990), 619-655.

12. Meise, R., Taylor, B.A., Vogt, D.: Extremal plurisubharmonic functions of linear growth on algebraic varieties, Math. Z. 219 (1995), 515-537.

13. Meise, R., Taylor, B.A., Vogt, D.: Continuous linear right inverses for partial differential operators of order 2 and fundamental solutions in half spaces, manuscr. math. 90 (1996), 449-464.

14. Meise, R., Taylor, B.A., Vogt, D.: Continuous linear right inverses for partial differential operators on non-quasianalytic classes and on ultradistributions, Math. Nachr. 180 (1996), 213-242.

15. Meise, R., Taylor, B.A., Vogt, D.: Phragmén-Lindelöf principles on algebraic varieties, J. Amer. Math. Soc. 11 (1998), 1-39.

16. Palamodov, V.P.: A criterion for splitness of differential complexes with constant coefficients, Geometrical and Algebraical Aspects in Several Complex Variables, C.A. Berenstein and D.C. Struppa (Eds.), EditEL (1991), 265-291.

17. Whitney, H.: Complex Analytic Varieties, Addison-Wesley, Reading-London 1972. 